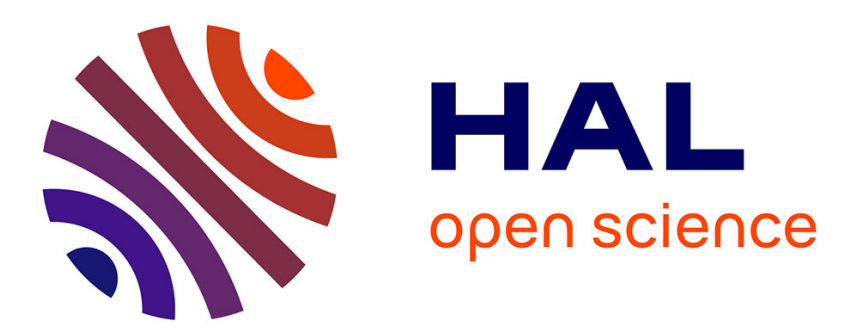

\title{
Vibrations of asymptotically and variationally based Uflyand-Mindlin plate models
}

\author{
I. Elishakoff, F. Hache, Noël Challamel
}

\section{To cite this version:}

I. Elishakoff, F. Hache, Noël Challamel. Vibrations of asymptotically and variationally based Uflyand-Mindlin plate models. International Journal of Engineering Science, 2017, 116, pp.58-73. 10.1016/j.ijengsci.2017.03.003 . hal-01693909

\section{HAL Id: hal-01693909 https://hal.science/hal-01693909}

Submitted on 31 Dec 2019

HAL is a multi-disciplinary open access archive for the deposit and dissemination of scientific research documents, whether they are published or not. The documents may come from teaching and research institutions in France or abroad, or from public or private research centers.
L'archive ouverte pluridisciplinaire HAL, est destinée au dépôt et à la diffusion de documents scientifiques de niveau recherche, publiés ou non, émanant des établissements d'enseignement et de recherche français ou étrangers, des laboratoires publics ou privés. 


\title{
Vibrations of asymptotically and variationally based Uflyand-Mindlin plate models
}

\author{
I. Elishakoff ${ }^{\mathrm{a}, *}$, F. Hache ${ }^{\mathrm{a}, \mathrm{b}}, \mathrm{N}$. Challamel ${ }^{\mathrm{b}}$ \\ a Department of Ocean and Mechanical Engineering, Florida Atlantic University, Boca Raton, FL 33481-0991, USA \\ ${ }^{\mathrm{b}}$ Université de Bretagne Sud (UBS), Institut de Recherche Dupuy de Lôme (IRDL), Centre de Recherche, Rue de Saint Maudé, BP92116, \\ 56321 Lorient cedex, France
}

In this paper, we provide alternative Uflyand-Mindlin's plate equations taking into account rotary inertia and shear deformation, based on both asymptotic expansion and variational arguments. The aim is to derive truncated versions of Uflyand-Mindlin's equations, specifically without the fourth order derivative term with respect to time. The truncated version of Uflyand-Mindlin's plate model may be derived starting from three-dimensional elasticity equations, by using asymptotic arguments based on expansion of displacements with respect to a small geometrical parameter. This expansion method also leads to a proper identification of the shear correction factor. It is shown that suitably modified variational derivation leads to an additional term which is shown to be negligible for determination of the fundamental natural frequency of the all-round simply supported plates, but may contribute significantly in estimation of higher natural frequencies. It is argued that the proposed version of Uflyand-Mindlin's plate equations is simpler and more consistent than the original Uflyand-Mindlin equations. Likewise, it is advantageous over the equation that stems from neglecting the fourth order time derivative in original Uflyand-Mindlin equations. The two alternative truncated models serve as intermediate theories between the classical plate theory and the original Uflyand-Mindlin theory their usefulness depending on the problem at hand.

\section{Introduction}

Initiated by Germain (1826) and corrected by Lagrange (1828), the Classical Plate Theory or German-Lagrange theory established the governing partial differential equations describing the mechanical behavior of thin plates in vibrations (Reismann, 1988). As explained by Ventsel and Krauthammer in their monograph (Ventsel \& Krauthammer, 2001). “Cauchy (1828) and Poisson (1829) were first to formulate the problem of plate bending on the basis of general equations of theory of elasticity". A few years later, Navier (1823) studied the theory for a flexural rigidity function of the thickness of the plate. Then, Kirchhoff (1850) brought many additional results about theory of thin plates. According to Leissa, in his forward to the book of Liew, Xiang, Kitipornchai, and Wang (1998), "a plate is typically considered to be thin when the ratio of its thickness to representative lateral dimension (e.g., circular plate diameter, square plate side length) is $1 / 20$ or less. In fact, most plates used in practical applications satisfy this criterion. This usually permits one to use classical thin plate theory

\footnotetext{
* Corresponding author.

E-mail addresses: elishako@fau.edu (I. Elishakoff), fhache2014@fau.edu (F. Hache), noel.challamel@univ-ubs.fr (N. Challamel).
} 
to obtain a fundamental (i.e., lowest) frequency with good accuracy". However, the classical plate theory may significantly overestimate higher frequencies. In the last century, lot of efforts have been made to describe the behavior of thick plates. As Liew et al. (1998), mention, Reissner (1944 1945) and Navier (1823) introduced "a theory of plates that takes account of shear deformation only" in addition to classical effects (see also Kirchhoff, 1850).

In 1921, Timoshenko (1921) published his study of vibrations of beams and introduced his governing differential equation that take into account both shear deformation and rotary inertia. The beam equations derived by Timoshenko are identical to the ones of Bresse (1859) that are corrected by a shear correction factor which may differ from unity. The Uflyand-Mindlin plate theory, also labelled as thick plate theory (Mindlin, 1951; Uflyand, 1948), constitutes an extension of the classical Kirchhoff-Love theory by taking into account shear deformation and rotary inertia and thus representing the two-dimensional analogue of the Bresse-Timoshenko beam theory.

The check on Google Scholar of the term "Uflyand-Mindlin Plate" yields 29500 hits attesting the enormous popularity of this theory. There is a definitive monograph devoted to Uflyand-Mindlin plates, by Liew et al. (1998). The inaccuracies described by Leissa (1969) are largely eliminated by use of the Uflyand-Mindlin theory, for it does include the effects of additional plate flexibility due to shear deformation, and additional plate inertia due to rotations (supplanting the translational inertia). Both effects decrease the frequencies. There are still other effects not accounted for by the Uflyand-Mindlin theory (e.g. stretching in the thickness direction, warping of the normal to the midplane), but these are typically unimportant for the lower frequencies until very thick plates are encountered. It appears instructive to quote Herrmann (1974): "Above all, Mindlin's work is motivated by a concern for physical reality. His analytical studies always begin with an intense desire to explain and interpret, in mathematical terms, observed but poorly understood physical phenomena".

Over the years, many researchers attempted to provide different derivation of Uflyand-Mindlin plate equations. One of them is based on an asymptotic approach considering a three-dimensional problem and reducing it to a two-dimensional problem (Vashakmadze, 1999). The use of asymptotic methods to validate a model has been used in the literature for beams (Berdichevsky \& Kvashina, 1974) and some attempt haves been performed for plates (Berdichevsky, 1973). Thus, Widera (1970), without any assumption about the displacements over the thickness of the plate and neglecting the effect of rotary inertia and shear deformations, derived a set of equations for the determination of the in-plane displacements, the same than for the classical thin plate theory. One of the aims of the present paper is to derive asymptotically a version of the Uflyand-Mindlin plate model through a power series expansion. In parallel of this approach, many articles have been published in the literature dedicated to the variational derivation of Uflyand-Mindlin's (Uflyand, 1948; Mindlin, 1951) plate equations.

Among them, one should mention the definitive monographs by Liew et al. (1998) or Wang, Reddy, and Lee (2000) and numerous references listed there (see for instance Brunelle \& Roberts, 1974; Brunelle, 1971; Sharma, Sharda, \& Nath, 2005). Elishakoff (1994) and Falsone, Settineri, and Elishakoff $(2014,2015)$ suggested to utilize truncated version of UflyandMindlin's (Mindlin, 1951) equation, neglecting the fourth order derivative in time. In this paper, we present a variational derivation of truncated Uflyand-Mindlin's equation based on slope inertia. It turns out that an additional term appears. We conduct comparison of four theories: (a) classical plate theory, (b) Uflyand-Mindlin's (Mindlin, 1951; Mindlin, Schacknow, \& Dereciewicz, 1956) original theory, (c) Elishakoff (1994) truncated set of equations, (d) variationally derived truncated set. Whereas we refrain from judging the superiority of the above methods, we emphasize that for lower range of frequencies the latter set at least leads to similar results in a much simpler formulation, in addition of being variationally derivable.

\section{Recapitulation of original and truncated Uflyand-Mindlin's theories}

\subsection{Original Uflyand-Mindlin plate theory via the equilibrium equations}

The plate is referred to a $x, y, z$-system of Cartesian coordinates. Assuming that the faces of the plate are under normal pressures $q_{1}$ and $q_{2}$, the boundary conditions are:

$$
\begin{aligned}
& \tau_{x z}\left(z= \pm \frac{h}{2}\right)=\tau_{y z}\left(z= \pm \frac{h}{2}\right)=0 \\
& \sigma_{z}\left(z=\frac{h}{2}\right)=-q_{1}(x, y, t) ; \sigma_{z}\left(z=-\frac{h}{2}\right)=-q_{2}(x, y, t)
\end{aligned}
$$

The bending and twisting moments and the transverse shearing forces are defined as follows:

$$
\left(\begin{array}{c}
M_{x} \\
M_{y} \\
M_{y x}
\end{array}\right)=\int_{-h / 2}^{h / 2}\left(\begin{array}{c}
\sigma_{x} \\
\sigma_{y} \\
\tau_{y x}
\end{array}\right) z d z ; \quad\left(\begin{array}{c}
Q_{x} \\
Q_{y}
\end{array}\right)=\int_{-h / 2}^{h / 2}\left(\begin{array}{c}
\tau_{x z} \\
\tau_{y z}
\end{array}\right) d z
$$

For an isotropic material one gets

$$
M_{x}=D\left(\Gamma_{x}+v \Gamma_{y}\right) ; M_{y}=D\left(\Gamma_{y}+v \Gamma_{x}\right) ; M_{y x}=\frac{D}{2}(1-v) \Gamma_{y x} ; Q_{x}=\kappa G h \Gamma_{x z} ; Q_{y}=\kappa G h \Gamma_{y z}
$$




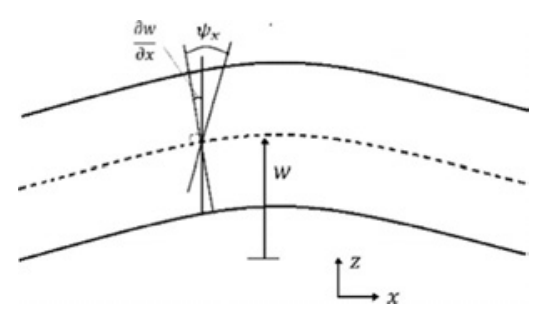

Fig. 1. Rotations of a transverse normal about the $y$ axis.

where $D=E h^{3} / 12\left(1-v^{2}\right)$ is the plate's flexural rigidity, $h$ the thickness of the plate, $v$ the Poisson's ratio, $\kappa$ the shear coefficient, $G$ the shear modulus of elasticity and $\Gamma_{x}, \Gamma_{y}, \Gamma_{y x}, \Gamma_{x z}, \Gamma_{y z}$ the plate-strains components defined as follows:

$$
\left(\Gamma_{x}, \Gamma_{y}, \Gamma_{y x}\right)=12 h^{-3} \int_{-\frac{h}{2}}^{\frac{h}{2}}\left(\begin{array}{c}
\varepsilon_{x} \\
\varepsilon_{y} \\
\gamma_{y x}
\end{array}\right) z d z ; \quad\left(\begin{array}{l}
\Gamma_{x z} \\
\Gamma_{y z}
\end{array}\right)=h^{-1} \int_{-\frac{h}{2}}^{\frac{h}{2}}\left(\begin{array}{c}
\gamma_{x z} \\
\gamma_{y z}
\end{array}\right) d z
$$

The usual plate-strain-displacement relationships are the following:

$$
\left(\begin{array}{c}
\varepsilon_{x} \\
\varepsilon_{y} \\
\gamma_{y x}
\end{array}\right)=\left(\begin{array}{c}
\frac{\partial u}{\partial x} \\
\frac{\partial v}{\partial y} \\
\frac{\partial v}{\partial x}+\frac{\partial u}{\partial y}
\end{array}\right) ;\left(\begin{array}{c}
\gamma_{x z} \\
\gamma_{y z}
\end{array}\right)=\left(\begin{array}{c}
\frac{\partial u}{\partial z}+\frac{\partial w}{\partial x} \\
\frac{\partial v}{\partial z}+\frac{\partial w}{\partial y}
\end{array}\right)
$$

In the Mindlin (1951) plate theory, the displacement components are assumed to be given by:

$$
u=z \psi_{x}(x, y, t) ; v=z \psi_{y}(x, y, t) ; w=w(x, y, t)
$$

$\psi_{x}$ and $\psi_{y}$ are the bending rotations of a transverse normal about the $x$ and $y$ axes, respectively, as shown in Fig. 1. It worth nothing that the Kirchhoff-Love plate theory can be recovered by setting $\psi_{x}=-\partial w / \partial x$ and $\psi_{y}=-\partial w / \partial y$.

Substituting Eq. (6) into Eq. (5) and then substituting in the resulting equation in Eq. (4), the plate-displacements components become:

$$
\Gamma_{x}=\frac{\partial \psi_{x}}{\partial x}, \Gamma_{y}=\frac{\partial \psi_{y}}{\partial y}, \Gamma_{y x}=\frac{\partial \psi_{y}}{\partial x}+\frac{\partial \psi_{x}}{\partial y}, \quad \Gamma_{x z}=\psi_{x}+\frac{\partial w}{\partial x}, \Gamma_{y z}=\psi_{y}+\frac{\partial w}{\partial y}
$$

Substitution of Eq. (7) into Eq. (3) leads to:

$$
\begin{aligned}
M_{x} & =D\left(\frac{\partial \psi_{x}}{\partial x}+v \frac{\partial \psi_{y}}{\partial y}\right) ; M_{y}=D\left(\frac{\partial \psi_{y}}{\partial y}+v \frac{\partial \psi_{x}}{\partial x}\right) ; M_{y x}=\frac{D}{2}(1-v)\left(\frac{\partial \psi_{y}}{\partial x}+\frac{\partial \psi_{x}}{\partial y}\right) \\
Q_{x} & =\kappa G h\left(\psi_{x}+\frac{\partial w}{\partial x}\right) ; Q_{y}=\kappa G h\left(\psi_{y}+\frac{\partial w}{\partial y}\right)
\end{aligned}
$$

The dynamic equilibrium equations of three-dimensional elasticity read:

$$
\begin{aligned}
& \frac{\partial \sigma_{x}}{\partial x}+\frac{\partial \tau_{y x}}{\partial y}+\frac{\partial \tau_{z x}}{\partial z}=\rho \frac{\partial^{2} u}{\partial t^{2}} \\
& \frac{\partial \tau_{y x}}{\partial x}+\frac{\partial \sigma_{y}}{\partial y}+\frac{\partial \tau_{z y}}{\partial z}=\rho \frac{\partial^{2} v}{\partial t^{2}} \\
& \frac{\partial \tau_{z x}}{\partial x}+\frac{\partial \tau_{y z}}{\partial y}+\frac{\partial \sigma_{z}}{\partial z}=\rho \frac{\partial^{2} w}{\partial t^{2}}
\end{aligned}
$$

Multiplication by $z$ and integration over the plate thickness provide, using Eq. (2), a system of three equations:

$$
\begin{aligned}
& \frac{\partial M_{x}}{\partial x}+\frac{\partial M_{y x}}{\partial y}-Q_{x}=\frac{\rho h^{3}}{12} \frac{\partial^{2} \psi_{x}}{\partial t^{2}} \\
& \frac{\partial M_{y x}}{\partial x}+\frac{\partial M_{y}}{\partial y}-Q_{y}=\frac{\rho h^{3}}{12} \frac{\partial^{2} \psi_{y}}{\partial t^{2}} \\
& \frac{\partial Q_{x}}{\partial x}+\frac{\partial Q_{y}}{\partial y}+q=\rho h \frac{\partial^{2} w}{\partial t^{2}}
\end{aligned}
$$


where $q=q_{2}-q_{1}$, is the resultant pressure. Substituting Eq. (8) into Eq. (10), the equations of motion become:

$$
\begin{aligned}
& \frac{D}{2}\left[(1-v) \nabla^{2} \psi_{x}+(1+v)\left(\frac{\partial^{2} \psi_{x}}{\partial x^{2}}+\frac{\partial^{2} \psi_{y}}{\partial x \partial y}\right)\right]-\kappa^{2} G h\left(\psi_{y}+\frac{\partial w}{\partial x}\right)=\frac{\rho h^{3}}{12} \frac{\partial^{2} \psi_{x}}{\partial t^{2}} \\
& \frac{D}{2}\left[(1-v) \nabla^{2} \psi_{y}+(1+v)\left(\frac{\partial^{2} \psi_{x}}{\partial x \partial y}+\frac{\partial^{2} \psi_{y}}{\partial y^{2}}\right)\right]-\kappa^{2} G h\left(\psi_{y}+\frac{\partial w}{\partial y}\right)=\frac{\rho h^{3}}{12} \frac{\partial^{2} \psi_{y}}{\partial t^{2}} \\
& \kappa^{2} G h\left(\nabla^{2} w+\frac{\partial \psi_{x}}{\partial x}+\frac{\partial \psi_{y}}{\partial y}\right)+q=\rho h \frac{\partial^{2} w}{\partial t^{2}}
\end{aligned}
$$

From Eq. (11), a governing equation of the deflection is obtained. Differentiating the two first equations of Eq. (11), with respect to $x$ and $y$, respectively, and adding these equations, one obtains, setting $\Phi=\partial \psi_{x} / \partial x+\partial \psi_{y} / \partial y$

$$
\left(D \nabla^{2}-\kappa^{2} G h-\frac{\rho h^{3}}{12} \frac{\partial^{2}}{\partial t^{2}}\right) \Phi=\kappa^{2} G h \nabla^{2} w
$$

where $\nabla^{2}$ is the Laplace operator. Substituting this equation in the first of the equations of motions Eq. (11) yields the governing differential equation which is the two-dimensional analogue of Timoshenko's beam equation

$$
\left(D \nabla^{2}-\frac{\rho h^{3}}{12} \frac{\partial^{2}}{\partial t^{2}}\right)\left(\nabla^{2}-\frac{\rho}{\kappa G} \frac{\partial^{2}}{\partial t^{2}}\right) w+\rho h \frac{\partial^{2} w}{\partial t^{2}}=\left(1-\frac{D \nabla^{2}}{\kappa G h}+\frac{\rho h^{3}}{12 K G} \frac{\partial^{2}}{\partial t^{2}}\right) q
$$

Without any external load, this equation is reduced to:

$$
D \nabla^{4}+\rho h \frac{\partial^{2} w}{\partial t^{2}}-\rho\left(\frac{h^{3}}{12}+\frac{D}{\kappa G}\right) \frac{\partial^{2}}{\partial t^{2}} \nabla^{2} w+\frac{\rho^{2} h^{3}}{12} \frac{1}{\kappa G} \frac{\partial^{4} w}{\partial t^{4}}=0
$$

or:

$$
D \nabla^{4} w+\rho h \frac{\partial^{2} w}{\partial t^{2}}-\rho \frac{h^{3}}{12}\left(1+\frac{12}{h^{3}} \frac{D}{\kappa G}\right) \frac{\partial^{2}}{\partial t^{2}} \nabla^{2} w+\frac{\rho^{2} h^{3}}{12} \frac{1}{\kappa G} \frac{\partial^{4} w}{\partial t^{4}}=0
$$

\subsection{Derivation of the original Uflyand--Mindlin plate model from the variational principle}

It appears instructive to provide the variational derivation of Uflyand-Mindlin's equation as presented by Mindlin (1951) himself and Liew et al. (1998). The potential energy is given by

$$
V=\iiint_{V} W d x d y d z
$$

where $V$ is the volume occupied by the plate, $W$ is the strain energy defined as follows:

$$
W=\frac{1}{2}\left(\sigma_{x} \varepsilon_{x}+\sigma_{y} \varepsilon_{y}+\sigma_{z} \varepsilon_{z}+\tau_{x y} \gamma_{x y}+\tau_{y z} \gamma_{y z}+\tau_{z x} \gamma_{z x}\right)
$$

Substitution of Eq. (5) into Eq. (17) yields

$$
2 W=\sigma_{x} \frac{\partial u}{\partial x}+\sigma_{y} \frac{\partial v}{\partial y}+\sigma_{z} \frac{\partial w}{\partial z}+\tau_{x y}\left(\frac{\partial v}{\partial x}+\frac{\partial u}{\partial y}\right)+\tau_{y z}\left(\frac{\partial w}{\partial y}+\frac{\partial v}{\partial z}\right)+\tau_{z x}\left(\frac{\partial w}{\partial x}+\frac{\partial u}{\partial z}\right)
$$

Defining the result of the integration of $W$ over thickness as $\bar{W}$ :

$$
\bar{W}=\int W d z
$$

Using Eqs. (6) and (19),

$$
2 \bar{W}=M_{x} \frac{\partial \psi_{x}}{\partial x}+M_{y} \frac{\partial \psi_{y}}{\partial y}+M_{y x}\left(\frac{\partial \psi_{y}}{\partial x}+\frac{\partial \psi_{x}}{\partial y}\right)+Q_{x}\left(\frac{\partial w}{\partial x}+\psi_{x}\right)+Q_{y}\left(\frac{\partial w}{\partial y}+\psi_{y}\right)
$$

or,

$$
2 \bar{W}=M_{x} \Gamma_{x}+M_{y} \Gamma_{y}+M_{y x} \Gamma_{y x}+Q_{x} \Gamma_{x z}+Q_{y} \Gamma_{y z}
$$

Substituting Eq. (8) into Eqs. (21) and (16), the potential energy in the following form is set as:

$$
\begin{aligned}
V= & \iint_{\Omega} \bar{W} d x d y=\iint_{\Omega} \frac{1}{2}\left(D\left\{\left(\frac{\partial \psi_{x}}{\partial x}+\frac{\partial \psi_{y}}{\partial y}\right)^{2}-2(1-v)\left[\frac{\partial \psi_{x}}{\partial x} \frac{\partial \psi_{y}}{\partial y}-\frac{1}{4}\left(\frac{\partial \psi_{x}}{\partial y}+\frac{\partial \psi_{y}}{\partial x}\right)^{2}\right]\right\}\right. \\
& \left.+\kappa G h\left[\left(\frac{\partial w}{\partial x}+\psi_{x}\right)^{2}+\left(\frac{\partial w}{\partial y}+\psi_{y}\right)^{2}\right]\right) d x d y
\end{aligned}
$$


The expression of the kinetic energy is the following:

$$
T=\iiint_{V} \frac{\rho}{2}\left[\left(\frac{\partial u}{\partial t}\right)^{2}+\left(\frac{\partial v}{\partial t}\right)^{2}+\left(\frac{\partial w}{\partial t}\right)^{2}\right] d v
$$

Using the expression of the displacement and integrating over the thickness

$$
T=\frac{1}{2} \iint_{\Omega} \rho h\left(\frac{\partial w}{\partial t}\right)^{2}+\frac{\rho h^{3}}{12}\left[\left(\frac{\partial \psi_{x}}{\partial t}\right)^{2}+\left(\frac{\partial \psi_{y}}{\partial t}\right)^{2}\right] d x d y
$$

where $\Omega$ is the area of the mid-surface of the plate.

According to the Hamilton's principle:

$$
\delta \int_{t_{i}}^{t} \Pi d t=0
$$

where the Lagrangian $\Pi$ is given by:

$$
\begin{aligned}
\Pi= & -V=\frac{1}{2} \iint_{\Omega}\left\{\frac{\rho h^{3}}{12}\left[\left(\frac{\partial \psi_{x}}{\partial t}\right)^{2}+\left(\frac{\partial \psi_{y}}{\partial t}\right)^{2}\right]+\rho h\left(\frac{\partial w}{\partial t}\right)^{2}\right\} d x d y \\
& -\frac{\kappa G h}{2} \iint_{\Omega}\left[\left(\frac{\partial w}{\partial x}+\psi_{x}\right)^{2}+\left(\frac{\partial w}{\partial y}+\psi_{y}\right)^{2}\right] d x d y \\
& -\frac{1}{2} \iint_{\Omega} \mathrm{D}\left\{\left(\frac{\partial \psi_{x}}{\partial x}+\frac{\partial \psi_{y}}{\partial y}\right)^{2}-2(1-v)\left[\frac{\partial \psi_{x}}{\partial x} \frac{\partial \psi_{y}}{\partial y}-\frac{1}{4}\left(\frac{\partial \psi_{x}}{\partial y}+\frac{\partial \psi_{y}}{\partial x}\right)^{2}\right]\right\} d x d y
\end{aligned}
$$

One obtains:

$$
\begin{aligned}
& \int_{t_{i}}^{t} \iint_{\Omega}\left\{-D\left[\left(\frac{\partial \psi_{x}}{\partial x}+v \frac{\partial \psi_{y}}{\partial y}\right) \frac{\partial \delta \psi_{x}}{\partial x}+\left(\frac{\partial \psi_{y}}{\partial y}+v \frac{\partial \psi_{x}}{\partial x}\right) \frac{\partial \delta \psi_{y}}{\partial y}\right]-\frac{D(1-v)}{2}\left(\frac{\partial \psi_{x}}{\partial y}+\frac{\partial \psi_{y}}{\partial x}\right)\left(\frac{\partial \delta \psi_{x}}{\partial y}+\frac{\partial \delta \psi_{y}}{\partial x}\right)\right. \\
& -\kappa G h\left[\left(\frac{\partial w}{\partial x}+\psi_{x}\right)\left(\frac{\partial \delta w}{\partial x}+\delta \psi_{x}\right)+\left(\frac{\partial w}{\partial y}+\psi_{y}\right)\left(\frac{\partial \delta w}{\partial y}+\delta \psi_{y}\right)\right]+\rho h \frac{\partial w}{\partial t} \frac{\partial \delta w}{\partial t} \\
& \left.\quad+\frac{\rho h^{3}}{12}\left(\frac{\partial \psi_{x}}{\partial t} \frac{\partial \delta \psi_{x}}{\partial t}+\frac{\partial \psi_{y}}{\partial t} \frac{\partial \delta \psi_{y}}{\partial t}\right)\right\} d x d y d t=0
\end{aligned}
$$

Integrating by part results in:

$$
\begin{aligned}
& \int_{t_{i}}^{t} \iint_{\Omega}\left\{D\left(\frac{\partial^{2} \psi_{x}}{\partial x^{2}} \delta \psi_{x}+\frac{\partial^{2} \psi_{y}}{\partial y^{2}} \delta \psi_{y}+v \frac{\partial^{2} \psi_{x}}{\partial x \partial y} \delta \psi_{y}+\mu \frac{\partial^{2} \psi_{y}}{\partial x \partial y} \delta \psi_{x}\right)\right. \\
& +\frac{D(1-v)}{2}\left(\frac{\partial^{2} \psi_{x}}{\partial y^{2}} \delta \psi_{x}+\frac{\partial^{2} \psi_{y}}{\partial x^{2}} \delta \psi_{y}+\frac{\partial^{2} \psi_{x}}{\partial x \partial y} \delta \psi_{y}+\frac{\partial^{2} \psi_{y}}{\partial x \partial y} \delta \psi_{x}\right) \\
& -\kappa G h\left[\left(\psi_{x} \delta \psi_{x}-\frac{\partial \psi_{x}}{\partial x} \delta w\right)+\left(\psi_{y} \delta \psi_{y}-\frac{\partial \psi_{y}}{\partial y} \delta w\right)+\left(\frac{\partial w}{\partial x} \delta \psi_{x}-\frac{\partial^{2} w}{\partial x^{2}} \delta w\right)\right. \\
& \left.\left.+\left(\frac{\partial w}{\partial y} \delta \psi_{y}-\frac{\partial^{2} w}{\partial y^{2}} \delta w\right)\right]-\rho h \frac{\partial^{2} w}{\partial t^{2}} \delta w-\frac{\rho h^{3}}{12}\left(\frac{\partial^{2} \psi_{x}}{\partial t^{2}} \delta \psi_{x}+\frac{\partial^{2} \psi_{y}}{\partial t^{2}} \delta \psi_{y}\right)\right\} d x d y d t \iint d x d y d t \\
& -\int_{t_{i}}^{t} \oint_{\Gamma}\left\{D\left[\left(\frac{\partial \psi_{x}}{\partial x}+v \frac{\partial \psi_{y}}{\partial y}\right) \delta \psi_{x} d y-\left(\frac{\partial \psi_{y}}{\partial y}+v \frac{\partial \psi_{x}}{\partial x}\right) \delta \psi_{y} d x\right]\right. \\
& +\frac{D(1-\mu)}{2}\left[\left(\delta \psi_{y}-\delta \psi_{x}\right) \frac{\partial \psi_{x}}{\partial y} d y-\left(\delta \psi_{x}-\delta \psi_{y}\right) \frac{\partial \psi_{y}}{\partial x} d x\right] \\
& \left.+\kappa G h\left[\left(\psi_{x}+\frac{\partial w}{\partial x}\right) d y-\left(\psi_{y}+\frac{\partial w}{\partial y}\right) d x\right] \delta w\right\} d t=0 .
\end{aligned}
$$




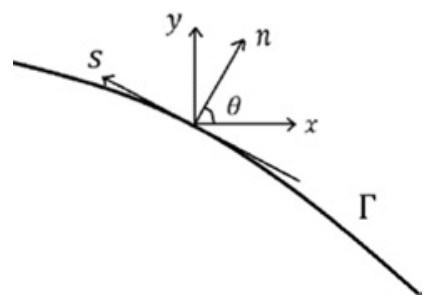

Fig. 2. Rectangular coordinates, and normal and tangential directions.

where $\Gamma$ is the boundary path. By grouping the terms in the foregoing functional with respect to the variation terms,

$$
\begin{aligned}
& \int_{t_{i}}^{t} \iint_{\Omega}\left\{\left[D\left(\frac{\partial^{2} \psi_{x}}{\partial x^{2}}+v \frac{\partial^{2} \psi_{y}}{\partial x \partial y}\right)+\frac{D(1-v)}{2}\left(\frac{\partial^{2} \psi_{x}}{\partial y^{2}}+v \frac{\partial^{2} \psi_{y}}{\partial x \partial y}\right)-\kappa G h\left(\psi_{x}+\frac{\partial w}{\partial x}\right)-\frac{\rho h^{3}}{12} \frac{\partial^{2} \psi_{x}}{\partial t^{2}}\right] \delta \psi_{x}\right. \\
& +\left[D\left(\frac{\partial^{2} \psi_{y}}{\partial y^{2}}+v \frac{\partial^{2} \psi_{x}}{\partial x \partial y}\right)+\frac{D(1-v)}{2}\left(\frac{\partial^{2} \psi_{y}}{\partial x^{2}}+v \frac{\partial^{2} \psi_{x}}{\partial x \partial y}\right)-\kappa G h\left(\psi_{y}+\frac{\partial w}{\partial y}\right)-\frac{\rho h^{3}}{12} \frac{\partial^{2} \psi_{y}}{\partial t^{2}}\right] \delta \psi_{y} \\
& \left.+\left[\kappa G h\left(\frac{\partial \psi_{x}}{\partial x}+\frac{\partial^{2} w}{\partial x^{2}}+\frac{\partial \psi_{y}}{\partial y}+\frac{\partial^{2} w}{\partial y^{2}}\right)-\rho h \frac{\partial^{2} w}{\partial t^{2}}\right] \delta w\right\} d x d y d t \\
& \quad-\int_{t_{i}}^{t} \oint_{\Gamma}\left[\left\{D\left(\frac{\partial \psi_{x}}{\partial x} d y+v \frac{\partial \psi_{y}}{\partial y} d y\right)-\frac{D(1-v)}{2}\left(\frac{\partial \psi_{x}}{\partial y} d x+\frac{\partial \psi_{y}}{\partial x} d x\right)\right\} \delta \psi_{x}\right. \\
& +\left\{-D\left(\frac{\partial \psi_{y}}{\partial y} d x+v \frac{\partial \psi_{x}}{\partial x} d x\right)+\frac{D(1-v)}{2}\left(\frac{\partial \psi_{x}}{\partial y} d y+\frac{\partial \psi_{y}}{\partial x} d y\right)\right\} \delta \psi_{y} \\
& \left.+\kappa G h\left(\psi_{x} d y+\frac{\partial w}{\partial x} d y-\psi_{y} d x-\frac{\partial w}{\partial y} d x\right) \delta w\right] d t=0
\end{aligned}
$$

Equating the coefficients of the variation terms to zero for the functional over the plate area, Eq. (11) are obtained, and thus, the governing differential equation is established variationally.

For boundary conditions, the line integral of Eq. (29) is set to zero and rewritten as:

$$
\begin{gathered}
\int_{t_{i}}^{t} \oint_{\Gamma}\left[D\left(\frac{\partial \psi_{x}}{\partial x}+v \frac{\partial \psi_{y}}{\partial y}\right) \delta \psi_{x} d y-D\left(\frac{\partial \psi_{y}}{\partial y}+v \frac{\partial \psi_{x}}{\partial x}\right) \delta \psi_{y} d x+\frac{D(1-v)}{2}\left(\frac{\partial \psi_{x}}{\partial y}+\frac{\partial \psi_{y}}{\partial x}\right) \delta \psi_{y} d y\right. \\
\left.-\frac{D(1-v)}{2}\left(\frac{\partial \psi_{x}}{\partial y}+\frac{\partial \psi_{y}}{\partial x}\right) \delta \psi_{x} d x+\kappa G h\left(\psi_{x}+\frac{\partial w}{\partial x}\right) \delta w d y-\kappa G h\left(\psi_{y}+\frac{\partial w}{\partial y}\right) \delta w d x\right] d t=0
\end{gathered}
$$

Substituting Eq. (8) into Eq. (30):

$$
\int_{t_{i}}^{t} \oint_{\Gamma}\left[M_{x x} \delta \psi_{x} d y-M_{y y} \delta \psi_{y} d x+M_{x y} \delta \psi_{y} d y-M_{x y} \delta \psi_{x} d x+Q_{x} \delta w d y-Q_{y} \delta w d x\right] d t=0
$$

According to the Frenet-Serret formulas, the subscripts $n$ and $s$ denoting the normal and tangential directions, respectively (see Fig. 2):

$$
\begin{array}{r}
d x=-\sin \theta d s ; d y=\cos \theta d s ; \psi_{x}=\psi_{n} \cos \theta-\psi_{s} \sin \theta ; \quad \psi_{y}=\psi_{n} \sin \theta+\psi_{s} \cos \theta ; Q_{n}=Q_{x} \cos \theta+Q_{y} \sin \theta \\
\quad M_{n n}=M_{x x} \cos ^{2} \theta+M_{y y} \sin ^{2} \theta+2 M_{x y} \sin \theta \cos \theta ; M_{n n}=\left(M_{y y}-M_{x x}\right) \sin \theta \cos \theta+M_{x y}\left(\cos ^{2} \theta-\sin ^{2} \theta\right)
\end{array}
$$

So, substituting Eq. (32) into Eq. (31), it becomes:

$$
\int_{t_{i}}^{t} \oint_{\Gamma}\left[M_{n n} \delta \psi_{n}+M_{n s} \delta \psi_{s}+Q_{n} \delta w\right] d s d t=0
$$

Hence, at the boundary of the plate:

$$
\begin{array}{ll}
M_{n n}=0 & \psi_{n} \\
M_{n s}=0 & \text { or } \psi_{s} \text { are specified } \\
Q_{n}=0 & w
\end{array}
$$

\subsection{Truncated Uflyand-Mindlin plate theory}

In his paper, Elishakoff (1994) stated that "the original Mindlin theory is inconsistent in the sense that it takes into account secondary effect of the interaction between the shear deformation and rotary inertia". Consequently, the last term 
in Eq. (15), the one with the fourth order derivative with respect to time, must not appear and he proposed to reduce Eq. (15) to:

$$
D \nabla^{4} w+\rho h \frac{\partial^{2} w}{\partial t^{2}}-\rho \frac{h^{3}}{12}\left(1+\frac{12}{h^{3}} \frac{D}{\kappa G}\right) \frac{\partial^{2}}{\partial t^{2}} \nabla^{2} w=0
$$

This truncated equation is directly derivable from equilibrium considerations, by replacing $\partial^{2} \psi_{x} / \partial t^{2}$ and $\partial^{2} \psi_{y} / \partial t^{2}$ in Eq. (11) by $\partial^{3} w / \partial x \partial t^{2}$ and $\partial^{3} w / \partial y \partial t^{2}$, respectively, as shown by Elishakoff et al. This process is an extension for plates of the one used by Elishakoff et al. (Elishakoff \& Livshits, 1984; Elishakoff \& Lubliner, 1985; Elishakoff et al., 2012; Elishakoff, Kaplunov, \& Nolde, 2015; Elishakoff, 2009) to obtain the truncated version of the Bresse-Timoshenko beam model.

Eq. (34) is also obtainable by asymptotic arguments from three-dimensional elasticity, following the work of Berdichevsky (1973) and Kaplunov (1996), using the reduction method, in which, the displacement is expanded in an infinite series of powers of the thickness coordinate (Widera, 1970) and approximate equations are derived, introducing an error that becomes smaller by increasing the order of the asymptotic expansion.

The three-dimensional equilibrium equations for a plate are written as follows (Widera, 1970):

$$
(\lambda+G)\left(\begin{array}{c}
\frac{\partial}{\partial x} \\
\frac{\partial}{\partial y} \\
\frac{\partial}{\partial z}
\end{array}\right)\left(\theta+\frac{\partial w}{\partial z}\right)+G\left(\nabla^{2}+\frac{\partial^{2}}{\partial z^{2}}\right)\left(\begin{array}{c}
u \\
v \\
w
\end{array}\right)=\rho \frac{\partial^{2}}{\partial t^{2}}\left(\begin{array}{c}
u \\
v \\
w
\end{array}\right)
$$

where $\lambda$ is the Lamé coefficient and $\theta$ and $\nabla^{2}$ are defined as

$$
\theta=\frac{\partial u}{\partial x}+\frac{\partial v}{\partial y} ; \nabla^{2}=\frac{\partial^{2}}{\partial x^{2}}+\frac{\partial^{2}}{\partial y^{2}}
$$

The stress vanishing on the free surfaces $z= \pm h / 2$

$$
\sigma_{z}\left(x, \frac{h}{2}\right)=\sigma_{z}\left(x,-\frac{h}{2}\right) ; \sigma_{y z}\left(x, \frac{h}{2}\right)+\sigma_{y z}\left(x,-\frac{h}{2}\right)=0
$$

The displacement solutions is developed in a power asymptotic expansion:

$$
\theta=\sum_{k=0}^{\infty} \theta_{k}(x, y, t) z^{k} ; w=\sum_{k=0}^{\infty} w_{k}(x, y, t) z^{k}
$$

Substituting in these equations, it becomes

$$
\begin{aligned}
& \sum_{n=1}^{\infty} 2(\lambda+2 G) n\left(\frac{h}{2}\right)^{2 n-1} w_{2 n}+\lambda\left(\frac{h}{2}\right)^{2 n-1} \theta_{2 n-1}=0 \\
& \nabla^{2} w_{0}+\sum_{n=1}^{\infty}\left\{\left(\frac{h}{2}\right)^{2 n} \nabla^{2} w_{2 n}+(2 n-1)\left(\frac{h}{2}\right)^{2 n-2} \theta_{2 n-1}\right\}=0 \\
& \left(G \nabla^{2}-\rho \frac{\partial^{2}}{\partial t^{2}}\right) w_{2 n}+(\lambda+2 G)(2 n+1)(2 n+2) w_{2 n+2}+(\lambda+G)(2 n+1) \theta_{2 n+1}=0 \\
& (\lambda+G) 2 n \nabla^{2} w_{2 n}+\left((\lambda+2 G) \nabla^{2}-\rho \frac{\partial^{2}}{\partial t^{2}}\right) \theta_{2 n-1}+G 2 n(2 n+1) \theta_{2 n+1}=0
\end{aligned}
$$

where $c^{2}=G / \rho$.

Consider the dimensionless variables:

$$
\bar{\theta}_{n}=L^{n} \theta_{n} ; \bar{w}_{n}=L^{n-1} w_{n} ; \bar{\nabla}^{2}=L^{2} \nabla^{2} ; \bar{h}=\frac{h}{2 L} ; \bar{t}=\frac{h t c}{2 L^{2}}=\frac{\bar{h} t c}{2 L}
$$

The three equations are re-expressed as:

$$
\begin{aligned}
& \sum_{n=0}^{\infty}\left[2(\lambda+2 G)(n+1) \bar{w}_{2 n+2}+\lambda \bar{\theta}_{2 n+1}\right] \bar{h}^{2 n}=0 \\
& \bar{\nabla}^{2} \bar{w}_{0}+\sum_{n=0}^{\infty}\left\{\bar{h}^{2} \bar{\nabla}^{2} \bar{w}_{2 n+2}+(2 n+1) \bar{\theta}_{2 n+1}\right\} \bar{h}^{2 n}=0 \\
& \bar{w}_{N+2}=-\frac{G}{(\lambda+2 G)(N+1)(N+2)}\left(\bar{\nabla}^{2}-\bar{h}^{2} \frac{\partial^{2}}{\partial \bar{t}^{2}}\right) \bar{w}_{N}-\frac{(\lambda+G)}{(\lambda+2 G)(N+2)} \bar{\theta}_{N+1} ; N=0,2,4,6, \ldots
\end{aligned}
$$




$$
\bar{\theta}_{N+3}=-\frac{(\lambda+G)}{G(N+3)} \bar{\nabla}^{2} \bar{w}_{N+2}-\frac{1}{(N+2)(N+3)}\left(\frac{(\lambda+2 G)}{G} \bar{\nabla}^{2}-\bar{h}^{2} \frac{\partial^{2}}{\partial \bar{t}^{2}}\right) \bar{\theta}_{N+1} ; N=0,2,4,6, \ldots
$$

At the fourth order

$$
\begin{aligned}
& 2(\lambda+2 G)\left(\bar{w}_{2}+2 \bar{w}_{4} \bar{h}^{2}+3 \bar{w}_{6} \bar{h}^{4}\right)+\lambda\left(\bar{\theta}_{1}+\bar{\theta}_{3} \bar{h}^{2}+\bar{\theta}_{5} \bar{h}^{4}\right)=0 \\
& \bar{\nabla}^{2}\left(\bar{w}_{0}+\bar{h}^{2} \bar{w}_{2}+\bar{h}^{4} \bar{w}_{4}\right)+\bar{\theta}_{1}+\bar{h}^{2} 3 \bar{\theta}_{3}+\bar{h}^{4} 5 \bar{\theta}_{5}=0 \\
& \bar{w}_{N+2}=-\frac{G}{(\lambda+2 G)(N+1)(N+2)}\left(\bar{\nabla}^{2}-\bar{h}^{2} \frac{\partial^{2}}{\partial \bar{t}^{2}}\right) \bar{w}_{N}-\frac{(\lambda+G)}{(\lambda+2 G)(N+2)} \bar{\theta}_{N+1} ; N=0,2,4,6, \ldots \\
& \bar{\theta}_{N+3}=-\frac{(\lambda+G)}{G(N+3)} \bar{\nabla}^{2} \bar{w}_{N+2}-\frac{1}{(N+2)(N+3)}\left(\frac{(\lambda+2 G)}{G} \bar{\nabla}^{2}-\bar{h}^{2} \frac{\partial^{2}}{\partial \bar{t}^{2}}\right) \bar{\theta}_{N+1} ; N=0,2,4,6, \ldots
\end{aligned}
$$

$\bar{w}_{i}(i=2,4,6)$ and $\bar{\theta}_{i}(i=3,5)$ are expressed with respect to $\theta_{1}$ and $\bar{w}_{0}$. Then, the two first equations are written in a matrix form:

$$
\left(\begin{array}{ll}
M_{11} & M_{12} \\
M_{21} & M_{22}
\end{array}\right)\left(\begin{array}{c}
\bar{w}_{0} \\
\theta_{1}
\end{array}\right)=\left(\begin{array}{l}
0 \\
0
\end{array}\right)
$$

where:

$$
\begin{aligned}
& M_{11}=\left\{-20(G-\lambda) \frac{\partial^{2}}{\partial \bar{t}^{2}} \bar{\nabla}^{2} \bar{h}^{4}+(2 G+3 \lambda) \bar{\nabla}^{6} \bar{h}^{4}+120(2 G+\lambda)\left(\frac{\partial^{2}}{\partial \bar{t}^{2}} \bar{h}^{2}-\bar{\nabla}^{2}\right)-20 \lambda \bar{\nabla}^{4} \bar{h}^{2}\right\}(2 G+\lambda) \\
& M_{12}=\left\{20(4 G+3 \lambda) \bar{\nabla}^{2} \bar{h}^{2}-120(2 G+\lambda)-20(3 G+2 \lambda) \frac{\partial^{2}}{\partial \bar{t}^{2}} \bar{h}^{4}-(6 G+5) \bar{\nabla}^{4} \bar{h}^{4}\right\}(2 G+\lambda) \\
& M_{21}=\left\{(2 G+3 \lambda) \bar{\nabla}^{4} \bar{h}^{4}+12 \lambda\left(\frac{\partial^{2}}{\partial \bar{t}^{2}} \bar{h}^{4}-\bar{\nabla}^{2} \bar{h}^{2}\right)-24(2 G+\lambda)\right\} \bar{\nabla}^{2} \\
& M_{22}=12(4 G+3 \lambda) \bar{\nabla}^{2} \bar{h}^{2}-12(2 G+\lambda) \frac{\partial^{2}}{\partial \bar{t}^{2}} \bar{h}^{4}-(6 G+5 \lambda) \bar{\nabla}^{4} \bar{h}^{4}-24(2 G+\lambda)
\end{aligned}
$$

In order to have a nontrivial solution, the determinant of the matrix has to vanish. It results in governing differential equations at different orders:

$\underline{\text { th } \text { order }}$

$$
(\lambda+G) \bar{\nabla}^{4} \bar{w}_{0}+\frac{3}{4}(\lambda+2 G) \frac{\partial^{2} \bar{w}_{0}}{\partial \bar{t}^{2}}=0
$$

2nd order

$$
-10(3 \lambda+4 G) \frac{\partial^{2}}{\partial \bar{t}^{2}} \bar{\nabla}^{2} \bar{h}^{2} \bar{w}_{0}-4(\lambda+G) \bar{\nabla}^{6} \bar{w}_{0} \bar{h}^{2}+20(\lambda+G) \bar{\nabla}^{4} \bar{w}_{0}+15(\lambda+2 G) \frac{\partial^{2} \bar{w}_{0}}{\partial \bar{t}^{2}}=0
$$

Or, in the dimensional form:

$\underline{\text { oth order }}$

$$
D \nabla^{4} \bar{w}_{0}+\rho h \frac{\partial^{2}}{\partial t^{2}} \bar{w}_{0}=0
$$

2nd order

$$
D \nabla^{4} \bar{w}_{0}+\rho h \frac{\partial^{2}}{\partial t^{2}} \bar{w}_{0}-\frac{2(2-v)}{(1-v)} \frac{\rho h^{3}}{12} \frac{\partial^{2}}{\partial t^{2}} \nabla^{2} \bar{w}_{0}-D \frac{1}{20} \nabla^{6} h^{2} \bar{w}_{0}=0
$$

It is seen that, at the zeroth order, Eq. (47) matches the governing differential equation of the thin plate theory. At this stage, the truncated Uflyand-Mindlin plate model is not obtainable from the three-dimensional elasticity equations. Indeed, an additional sixth order spatial derivative term occurs in the governing differential equation.

$$
\left[5-\bar{\nabla}^{2} \bar{h}^{2}\right] 4(\lambda+G) \bar{\nabla}^{4} \bar{w}_{0}-10(3 \lambda+4 G) \bar{h}^{2} \frac{\partial^{2}}{\partial \bar{t}^{2}} \bar{\nabla}^{2} \bar{w}_{0}+15(\lambda+2 G) \frac{\partial^{2} \bar{w}_{0}}{\partial \bar{t}^{2}}=0
$$

In fact, multiplying by operator $\left[1+\left(\bar{h}^{2} / 5\right) \bar{\nabla}^{2}\right]$ and neglecting the terms of order $\bar{h}^{4}$ leads to:

$$
20(\lambda+G) \bar{\nabla}^{4} \bar{w}_{0}+[3(\lambda+2 G)-10(3 \lambda+4 G)] \bar{h}^{2} \frac{\partial^{2}}{\partial \bar{t}^{2}} \bar{\nabla}^{2} \bar{w}_{0}+15(\lambda+2 G) \frac{\partial^{2} \bar{w}_{0}}{\partial \bar{t}^{2}}=0
$$

Thus, the equation is reduced to a fourth order space derivative governing differential equation.

$$
D \nabla^{4} \bar{w}_{0}+\rho h \frac{\partial^{2}}{\partial t^{2}} \bar{w}_{0}-\frac{\rho h^{3}}{12}\left[1+\frac{12 D}{h^{3} G} \frac{6-v}{5}\right] \frac{\partial^{2}}{\partial t^{2}} \nabla^{2} \bar{w}_{0}=0
$$


The equation is reduced to the truncated version of Uflyand-Mindlin plate theory with a shear coefficient equal to

$$
\kappa=\frac{5}{6-v}
$$

This shear coefficient coincides with the one found by Hutchinson (1984), Goldenveizer, Kaplunov, and Nolde (1993) and Stephen (1997) for plate models. By replacing $v$ by $v /(1+v)$, one also obtains $\kappa=5(1+v) /(1+v)$ which is the value reported by Stephen (1981) and more recently by Elishakoff et al. (2015) for beams (under plane stress assumptions). Kaneko (1975) also commented on this value and mentioned that already Timoshenko (1922) implicitly used this formulae.

In other words, the truncated Mindlin plate theory, as for the Kirchhoff-Love theory, is asymptotically consistent. Kirchhoff-Love theory is the zeroth order approximation whereas the truncated Uflyand-Mindlin plate model may be considered as the second-order asymptotically derived theory.

\section{Variational derivation of Uflyand-Mindlin plate theory based on slope inertia}

One of the aims of this paper is to extend Elishakoff (2009) analysis to truncated Uflyand-Mindlin plates, in variational setting, and to compare the different versions of the Uflyand-Mindlin plate model in order to establish the potential superiority on one of them on the others.

In his paper, Mindlin (1951) uses the exact expression of the kinetic energy in three dimensions given by the general linear theory of elasticity. By using Eq. (6) which contains also a correction in order to take into account the shear effect, Mindlin "overcorrected", as it were, the kinetic energy. It is suggested in this paper to replace the expression of the kinetic energy given in Eq. (24) by:

$$
T=\frac{1}{2} \iint \frac{\rho h^{3}}{12}\left[\left(\frac{\partial^{2} w}{\partial t \partial x}\right)^{2}+\left(\frac{\partial^{2} w}{\partial t \partial y}\right)^{2}\right]+\rho h\left(\frac{\partial w}{\partial t}\right)^{2} d x d y
$$

Note that Eq. (53) represents generalization of Bresse (1859) and Rayleigh (1877) beam equation.

Using Hamilton's principle in conjunction with Eq. (53) rather than Eq. (24) yields to in new circumstances, expressions of plates:

$$
\begin{aligned}
& \int_{t_{i}}^{t} \iint_{\Omega}\left\{-D\left(\frac{\partial \psi_{x}}{\partial x} \frac{\partial \delta \psi_{x}}{\partial x}+\frac{\partial \psi_{y}}{\partial y} \frac{\partial \delta \psi_{y}}{\partial y}+v \frac{\partial \psi_{x}}{\partial x} \frac{\partial \delta \psi_{y}}{\partial y}+v \frac{\partial \delta \psi_{x}}{\partial x} \frac{\partial \psi_{y}}{\partial y}\right)-\frac{D(1-v)}{2}\left(\frac{\partial \psi_{x}}{\partial y}+\frac{\partial \psi_{y}}{\partial x}\right)\right. \\
& \quad\left(\frac{\partial \delta \psi_{x}}{\partial y}+\frac{\partial \delta \psi_{y}}{\partial x}\right)-\kappa^{2} G h\left[\left(\frac{\partial w}{\partial x}+\psi_{x}\right)\left(\frac{\partial \delta w}{\partial x}+\delta \psi_{x}\right)+\left(\frac{\partial w}{\partial y}+\psi_{y}\right)\left(\frac{\partial \delta w}{\partial y}+\delta \psi_{y}\right)\right]+\rho h \frac{\partial w}{\partial t} \frac{\partial \delta w}{\partial t} \\
& \left.+\frac{\rho h^{3}}{12}\left(\frac{\partial^{2} w}{\partial t \partial x} \frac{\partial^{2} \delta w}{\partial t \partial x}+\frac{\partial^{2} w}{\partial t \partial y} \frac{\partial^{2} \delta w}{\partial t \partial t y}\right)\right\} d x d y d t=0
\end{aligned}
$$

Integrating by part,

$$
\begin{aligned}
& \int_{t_{i}}^{t} \iint_{\Omega}\left\{D\left(\frac{\partial^{2} \psi_{x}}{\partial x^{2}} \delta \psi_{x}+\frac{\partial^{2} \psi_{y}}{\partial y^{2}} \delta \psi_{y}+v \frac{\partial^{2} \psi_{x}}{\partial x \partial y} \delta \psi_{y}+v \frac{\partial^{2} \psi_{y}}{\partial x \partial y} \delta \psi_{x}\right)\right. \\
& +\frac{D(1-v)}{2}\left(\frac{\partial^{2} \psi_{x}}{\partial y^{2}} \delta \psi_{x}+\frac{\partial^{2} \psi_{y}}{\partial x^{2}} \delta \psi_{y}+\frac{\partial^{2} \psi_{x}}{\partial x \partial y} \delta \psi_{y}+\frac{\partial^{2} \psi_{y}}{\partial x \partial y} \delta \psi_{x}\right) \\
& -\kappa G h\left[\left(\psi_{x} \delta \psi_{x}-\frac{\partial \psi_{x}}{\partial x} \delta w\right)+\left(\psi_{y} \delta \psi_{y}-\frac{\partial \psi_{y}}{\partial y} \delta w\right)+\left(\frac{\partial w}{\partial x} \delta \psi_{x}-\frac{\partial^{2} w}{\partial x^{2}} \delta w\right)\right. \\
& \left.\left.+\left(\frac{\partial w}{\partial y} \delta \psi_{y}-\frac{\partial^{2} w}{\partial y^{2}} \delta w\right)\right]-\rho h \frac{\partial^{2} w}{\partial t^{2}} \delta w+\frac{\rho h^{3}}{12} \frac{\partial^{2}}{\partial t^{2}} \nabla^{2} w \delta w\right\} d x d y d t \\
& -\int_{t_{i}}^{t} \oint_{\Gamma}\left\{D\left(\frac{\partial \psi_{x}}{\partial x} \delta \psi_{x} d y-\frac{\partial \psi_{y}}{\partial y} \delta \psi_{y} d x-v \frac{\partial \psi_{x}}{\partial x} \delta \psi_{y} d x+v \frac{\partial \psi_{y}}{\partial y} \delta \psi_{x} d y\right)\right. \\
& +\frac{D(1-v)}{2}\left(-\frac{\partial \psi_{x}}{\partial y} \delta \psi_{x} d y+\frac{\partial \psi_{x}}{\partial y} \delta \psi_{y} d y-\frac{\partial \psi_{y}}{\partial x} \delta \psi_{x} d x+\frac{\partial \psi_{y}}{\partial x} \delta \psi_{y} d x\right) \\
& \left.+\kappa G h\left(\psi_{x} d y+\frac{\partial w}{\partial x} d y-\psi_{y} d x-\frac{\partial w}{\partial y} d x\right) \delta w+\frac{\rho h^{3}}{12}\left(\frac{\partial^{2} w}{\partial t^{2} \partial x} d y-\frac{\partial^{2} w}{\partial t^{2} \partial y} d x\right) \delta w\right\} d t=0
\end{aligned}
$$

By grouping the terms in the foregoing functional with respect to the variation terms, it derives,

$$
\int_{t_{i}}^{t} \iint_{\Omega}\left\{\left[D\left(\frac{\partial^{2} \psi_{x}}{\partial x^{2}}+v \frac{\partial^{2} \psi_{y}}{\partial x \partial y}\right)+\frac{D(1-v)}{2}\left(\frac{\partial^{2} \psi_{x}}{\partial y^{2}}+v \frac{\partial^{2} \psi_{y}}{\partial x \partial y}\right)-\kappa G h\left(\psi_{x}+\frac{\partial w}{\partial x}\right)\right] \delta \psi_{x}\right.
$$




$$
\begin{aligned}
& +\left[D\left(\frac{\partial^{2} \psi_{y}}{\partial y^{2}}+v \frac{\partial^{2} \psi_{x}}{\partial x \partial y}\right)+\frac{D(1-v)}{2}\left(\frac{\partial^{2} \psi_{y}}{\partial x^{2}}+v \frac{\partial^{2} \psi_{x}}{\partial x \partial y}\right)-\kappa G h\left(\psi_{y}+\frac{\partial w}{\partial y}\right)\right] \delta \psi_{y} \\
& \left.+\left[\kappa G h\left(\frac{\partial \psi_{x}}{\partial x}+\frac{\partial^{2} w}{\partial x^{2}}+\frac{\partial \psi_{y}}{\partial y}+\frac{\partial^{2} w}{\partial y^{2}}\right)-\rho h \frac{\partial^{2} w}{\partial t^{2}}+\frac{\rho h^{3}}{12} \frac{\partial^{2}}{\partial t^{2}} \nabla^{2} w\right] \delta w\right\} d x d y d t \\
& -\int_{t_{i}}^{t} \oint_{\Gamma}\left[\left\{D\left(\frac{\partial \psi_{x}}{\partial x} d y+v \frac{\partial \psi_{y}}{\partial y} d y\right)-\frac{D(1-v)}{2}\left(\frac{\partial \psi_{x}}{\partial y} d x+\frac{\partial \psi_{y}}{\partial x} d x\right)\right\} \delta \psi_{x}\right. \\
& +\left\{-D\left(\frac{\partial \psi_{y}}{\partial y} d x+v \frac{\partial \psi_{x}}{\partial x} d x\right)+\frac{D(1-v)}{2}\left(\frac{\partial \psi_{x}}{\partial y} d y+\frac{\partial \psi_{y}}{\partial x} d y\right)\right\} \delta \psi_{x} \\
& \left.+\kappa G h\left(\psi_{x} d y+\frac{\partial w}{\partial x} d y-\psi_{y} d x-\frac{\partial w}{\partial y} d x\right) \delta w-\frac{\rho h^{3}}{12}\left(\frac{\partial^{2} w}{\partial t^{2} \partial x} d y-\frac{\partial^{2} w}{\partial t^{2} \partial y} d x\right) \delta w\right] d t=0
\end{aligned}
$$

Equating the coefficients of the variation terms to zero for the functional over the plate area, the equations of motion are obtained as follows,

$$
\begin{aligned}
& \frac{D}{2}\left[(1-v) \nabla^{2} \psi_{x}+(1+v) \frac{\partial \Phi}{\partial x}\right]-\kappa G h\left(\psi_{x}+\frac{\partial w}{\partial x}\right)=0 \\
& \frac{D}{2}\left[(1-v) \nabla^{2} \psi_{y}+(1+v) \frac{\partial \Phi}{\partial y}\right]-\kappa G h\left(\psi_{y}+\frac{\partial w}{\partial y}\right)=0 \\
& \kappa G h\left(\nabla^{2} w+\Phi\right)+q=\rho h\left(1-\frac{h^{2}}{12} \nabla^{2}\right) \frac{\partial^{2} w}{\partial t^{2}}
\end{aligned}
$$

From Eqs. (57), a governing equation of the deflection is obtained. Differentiating Eqs. (57a) and (57b) with respect to $x$ and $y$, respectively, and adding these equations, one obtains

$$
\left(D \nabla^{2}-\kappa G h\right) \Phi=\kappa G h \nabla^{2} w
$$

According to Eq. (57b):

$$
\Phi=\frac{\rho}{\kappa G}\left(1-\frac{h^{2}}{12} \nabla^{2}\right) \frac{\partial^{2} w}{\partial t^{2}}-\frac{q}{\kappa G h}-\nabla^{2} w
$$

Substituting this equation in Eq. (58), one obtains the governing differential equation

$$
\left(\kappa^{2} G h-D \nabla^{2}\right)\left[\frac{\rho}{\kappa G}\left(1-\frac{h^{2}}{12} \nabla^{2} w\right) \frac{\partial^{2} w}{\partial t^{2}}-\frac{q}{\kappa G h}-\nabla^{2} w\right]=-\kappa^{2} G h \nabla^{2} w
$$

Setting $q=0$ for free vibrations leads to

$$
D \nabla^{4} w+\rho h \frac{\partial^{2} w}{\partial t^{2}}-\frac{\rho h^{3}}{12}\left(1+\frac{12}{h^{3}} \frac{D}{\kappa G}\right) \frac{\partial^{2}}{\partial t^{2}} \nabla^{2} w+\frac{\rho h^{2} D}{12 \kappa G} \frac{\partial^{2}}{\partial t^{2}} \nabla^{4} w=0
$$

The difference with original Uflyand-Mindlin equations is two-fold (a) an additional, underlined, term occurs, and (b) the fourth-order time derivative does not appear.

For boundary conditions, the line integral of Eq. (56) is set to zero and rewritten as:

$$
\begin{aligned}
& \int_{t_{i}}^{t} \oint_{\Gamma}\left[\left\{D\left(\frac{\partial \psi_{x}}{\partial x}+v \frac{\partial \psi_{y}}{\partial y}\right) d y-\frac{D(1-v)}{2}\left(\frac{\partial \psi_{x}}{\partial y}+\frac{\partial \psi_{y}}{\partial x}\right) d x\right\} \delta \psi_{x}\right. \\
& \quad+\left\{-D\left(\frac{\partial \psi_{y}}{\partial y}+v \frac{\partial \psi_{x}}{\partial x}\right) d x+\frac{D(1-v)}{2}\left(\frac{\partial \psi_{x}}{\partial y}+\frac{\partial \psi_{y}}{\partial x}\right) d y\right\} \delta \psi_{y} \\
& \left.\quad+\left[\left(\frac{\rho h^{2}}{12} \frac{\partial^{2} w}{\partial t^{2} \partial x}+\kappa G \psi_{x}+\kappa G \frac{\partial w}{\partial x}\right) d y-\left(\frac{\rho h^{2}}{12} \frac{\partial^{2} w}{\partial t^{2} \partial y}+\kappa G \psi_{y}+\kappa G \frac{\partial w}{\partial y}\right) d x\right] h \delta w\right] d t=0
\end{aligned}
$$

Eq. (62) implies that along the boundary of the plate. Setting $\tilde{Q}_{x}$ and $\tilde{Q}_{y}$

$$
\tilde{Q}_{x}=\kappa G h\left(\psi_{x}+\frac{\partial w}{\partial x}\right)-\frac{\rho h^{3}}{12} \frac{\partial^{2} w}{\partial t^{2} \partial x} ; \quad \tilde{Q}_{y}=\kappa G h\left(\psi_{y}+\frac{\partial w}{\partial y}\right)+\frac{\rho h^{3}}{12} \frac{\partial^{2} w}{\partial t^{2} \partial y}
$$

Hence, at the boundary of the plate, following the original Bresse-Timoshenko model:

$$
\begin{aligned}
& M_{n n}=0 \quad \psi_{n} \\
& M_{n s}=0 \text { or } \psi_{s} \text { are specified } \\
& \tilde{Q}_{n}=0 \quad w
\end{aligned}
$$




\section{All-round simply supported Uflyand-Mindlin plate: comparison of four versions}

The aim of this section is to use the same approach as Timoshenko (1921) and to evaluate the contribution of the all terms in the governing differential equation. Vibrations of all round simply supported Uflyand-Mindlin plate was studied by Mindlin et al. (1956) and Wang (1994). Hereinafter, the paper by Wang (1994), who thoroughly investigated vibrations of simply supported Uflyand-Mindlin plates is partially followed here. The boundary conditions considering the Uflyand-Mindlin plate model based on slope inertia are the same than the original Uflyand-Mindlin plate model and the truncated Uflyand-Mindlin plate model. First of all, consider the following nondimensional quantities:

$$
\xi=\frac{x}{a} ; \quad \eta=\frac{y}{b} ; \quad \beta=\frac{h}{b} ; \bar{w}=\frac{w}{b} ; \quad \chi=\frac{a}{b} ; \lambda=\frac{\omega^{2} b^{4} \rho h}{D} ; \bar{\Pi}=\frac{2 \Pi b}{a D}
$$

The nondimensional Lagrangian can be written as follows:

$$
\begin{aligned}
\bar{\Pi}= & \int_{0}^{1} \int_{0}^{1}\left(\left(\frac{1}{\chi} \frac{\partial \psi_{x}}{\partial \xi}+\frac{\partial \psi_{y}}{\partial \eta}\right)^{2}-2(1-v)\left[\frac{1}{\chi} \frac{\partial \psi_{x}}{\partial \xi} \frac{\partial \psi_{y}}{\partial \eta}-\frac{1}{4}\left(\frac{\partial \psi_{x}}{\partial \eta}+\frac{1}{\chi} \frac{\partial \psi_{y}}{\partial \xi}\right)^{2}\right]\right. \\
& +\frac{6(1-v) \kappa}{\beta^{2}}\left[\left(\frac{1}{\chi} \frac{\partial \bar{w}}{\partial \xi}+\psi_{x}\right)^{2}+\left(\frac{\partial \bar{w}}{\partial \eta}+\psi_{y}\right)^{2}\right] \\
& \left.-\lambda\left\{\frac{\beta^{2}}{12}\left[\gamma_{2}\left(\frac{1}{\chi} \frac{\partial \bar{w}}{\partial \xi}\right)^{2}+\gamma_{2}\left(\frac{\partial \bar{w}}{\partial \eta}\right)^{2}+\gamma_{1} \psi_{x}^{2}+\gamma_{1} \psi_{y}^{2}\right]+\bar{w}^{2}\right\}\right) d \xi d \eta
\end{aligned}
$$

where the control parameters $\gamma_{1}$ and $\gamma_{2}$ are introduced, with $\left(\gamma_{1}, \gamma_{2}\right)$ equals to $(1,0)$ and $(0,1)$ for the original Mindlin plate theory and the Mindlin model based on slope inertia, respectively.

For a simply supported plate, the Navier's approach (Navier, 1823) is used with exact mode shapes (Wang, 1994):

$$
\psi_{x}=A_{m n} \cos (m \pi \xi) \sin (n \pi \eta) ; \psi_{y}=B_{m n} \sin (m \pi \xi) \cos (n \pi \eta) ; \bar{w}=C_{m n} \sin (m \pi \xi) \sin (n \pi \eta)
$$

in which $A_{m n}, B_{m n}$ and $C_{m n}$ are coefficients.

Substituting Eq. (66) into Eq. (65) one gets:

$$
\begin{aligned}
\bar{\Pi}= & \frac{1}{4}\left(\frac{m \pi}{\chi} A_{m n}+n \pi B_{m n}\right)^{2}-\frac{(1-v)}{2}\left[\frac{m n \pi^{2}}{\chi} A_{m n} B_{m n}-\frac{1}{4}\left(n \pi A_{m n}+\frac{m \pi}{\chi} B_{m n}\right)^{2}\right] \\
& +\frac{6(1-v) \kappa}{4 \beta^{2}}\left[\left(\frac{m \pi}{\chi} C_{m n}+A_{m n}\right)^{2}+\left(n \pi C_{m n}+B_{m n}\right)^{2}\right] \\
& -\frac{\lambda}{4}\left\{\gamma_{2} C_{m n}^{2} \frac{\beta^{2}}{12}\left[\left(\frac{m \pi}{\chi}\right)^{2}+(n \pi)^{2}\right]+\gamma_{1}\left(A_{m n}^{2}+B_{m n}^{2}\right) \frac{\beta^{2}}{12}+C_{m n}^{2}\right\}
\end{aligned}
$$

According to the principle of minimum of energy, the coefficients $A_{m n}, B_{m n}$ and $C_{m n}$ minimize $\bar{\Pi}$ :

$$
\frac{\partial \bar{\Pi}}{\partial A_{m n}}=\frac{\partial \bar{\Pi}}{\partial B_{m n}}=\frac{\partial \bar{\Pi}}{\partial C_{m n}}=0
$$

Substitution of Eq. (67) into Eq. (68) yields a set of homogeneous equations, given under the matrix form:

$$
\left(\begin{array}{ccc}
K_{1}-\gamma \lambda I & K_{2} & K_{3} \\
& K_{4}-\gamma_{1} \lambda I & K_{5} \\
\text { symmetric } & & K_{6}-\gamma\left(\lambda_{2} K_{7}+1\right)
\end{array}\right)\left(\begin{array}{l}
A_{m n} \\
B_{m n} \\
C_{m n}
\end{array}\right)=0
$$

in which the coefficients $I$ and $K_{i}$ are defined as follows:

$$
\begin{aligned}
I & =\frac{\beta^{2}}{12} ; K_{1}=\sqrt{\lambda_{m n}}-\frac{(1+v)}{2} n^{2} \pi^{2}+\frac{6(1-v) \kappa}{\beta^{2}} ; K_{2}=\frac{(1+v)}{2} \frac{m n \pi^{2}}{\chi} \\
K_{3} & =\frac{6(1-v) \kappa}{\beta^{2}} \frac{m \pi}{\chi} ; K_{4}=\sqrt{\lambda_{m n}}-\frac{(1+v)}{2 \chi^{2}} m^{2} \pi^{2}+\frac{6(1-v) \kappa}{\beta^{2}} \\
K_{5} & =\frac{6(1-v) \kappa}{\beta^{2}} n \pi ; K_{6}=\frac{6(1-v) \kappa}{\beta^{2}} \sqrt{\lambda_{m n}} ; K_{7}=\frac{\beta^{2}}{12} \sqrt{\lambda_{m n}}
\end{aligned}
$$

where $\lambda_{m n}$ is the nondimensional frequency obtained from the classical plate theory (Leissa, 1969; Wang, 1994; Reddy, 2006)

$$
\lambda_{m n}=\pi^{4}\left(n^{2}+\frac{m^{2}}{\chi^{2}}\right)^{2}
$$


In order to have a nontrivial solution to Eq. (69), the determinant of the system must vanish.

For the original Uflyand-Mindlin plate theory, $\left(\gamma_{1}, \gamma_{2}\right)=(1,0)$, and Wang (1994) obtained a cubic characteristic equation, for unknown $\lambda$

$$
\begin{aligned}
& \operatorname{det}\left(\begin{array}{ccc}
K_{1}-\gamma_{1} \lambda I & K_{2} & K_{3} \\
\text { symmetric } & K_{4}-\gamma_{1} \lambda I & K_{5} \\
\left(K_{1}-\lambda I\right)\left(K_{4}-\lambda I\right)\left(K_{6}-\lambda\right)+2 K_{2} K_{5} K_{3}-\left(K_{6}-\lambda\right) K_{2}^{2}-\left(K_{1}-\lambda I\right) K_{5}^{2}-\left(K_{4}-\lambda I\right) K_{3}^{2}=0
\end{array}\right.
\end{aligned}
$$

The root that furnishes the lowest eigenvalue is given by.

$$
\lambda=36 \frac{(1-v) \kappa}{\beta^{4}}\left\{\left[1+\frac{\beta^{2} \sqrt{\lambda_{m n}}}{12}\left(1+\frac{2}{(1-v) \kappa}\right)\right]-\sqrt{\left[1+\frac{\beta^{2} \sqrt{\lambda_{m n}}}{12}\left(1+\frac{2}{(1-v) \kappa}\right)\right]^{2}-\frac{\beta^{4} \lambda_{m n}}{18(1-v) \kappa}}\right\}
$$

With the Uflyand-Mindlin equation based on slope inertia, one obtains a linear equation of unknown $\lambda$

$$
\begin{aligned}
& \operatorname{det}\left(\begin{array}{ccc}
K_{1} & K_{2} & K_{3} \\
& K_{4} & K_{5} \\
\text { symmetric } & & K_{6}-\lambda\left(K_{7}+1\right)
\end{array}\right)=0 \\
& K_{1} K_{4}\left[K_{6}-\lambda\left(K_{7}+1\right)\right]+2 K_{1} K_{1} K_{1}-\left[K_{6}-\lambda\left(K_{7}+1\right)\right] K_{2}^{2}-K_{1} K_{5}^{2}-K_{4} K_{3}^{2}=0
\end{aligned}
$$

It leads to a single root

$$
\lambda=\frac{K_{1} K_{4} K_{6}+2 K_{2} K_{3} K_{5}-K_{3}^{2} K_{4}-K_{1} K_{5}^{2}-K_{2}^{2} K_{6}}{K_{1} K_{4} K_{7}-K_{2}^{2} K_{7}}
$$

Substituting Eq. (66) into Eq. (34), Elishakoff (1994) expressed the natural frequency as follows:

$$
\omega^{2}=\frac{\frac{D}{\rho h}\left(\frac{m^{2} \pi^{2}}{a^{2}}+\frac{n^{2} \pi^{2}}{b^{2}}\right)^{2}}{1+\left(\frac{D}{\kappa G h}+\frac{h^{2}}{12}\right)\left(\frac{m^{2} \pi^{2}}{a^{2}}+\frac{n^{2} \pi^{2}}{b^{2}}\right)}
$$

Using the notations of this paper, Eq. (76) can be rewritten as follows:

$$
\lambda=\frac{\pi^{4}\left(\frac{m^{2}}{\chi^{2}}+n^{2}\right)^{2}}{1+\frac{1}{12}\left(\frac{2}{\kappa(1-v)}+1\right) \beta^{2} \pi^{2}\left(\frac{m^{2}}{\chi^{2}}+n^{2}\right)}
$$

The nondimensional frequencies are obtained in Table 1 , fixing constants at values $\kappa=\pi^{2} / 12, \nu=0.3$ and $\beta=0.2$.

Furthermore, Wittrick (1987) provided the analytical, three-dimensional solutions for a square plate:

$$
\mathrm{Y}^{2} \lambda^{2}=4(1-\mathrm{Y} \lambda)\left\{\frac{\sqrt{\left(1-\frac{1-2 v}{2-2 \nu} \mathrm{Y} \lambda\right)} \tanh \left[\left(\frac{\beta \pi}{2 \chi}\right) \sqrt{\left.\left(m^{2}+\chi^{2} n^{2}\right)(1-\mathrm{Y} \lambda)\right]}\right.}{\sqrt{(1-\mathrm{Y} \lambda)} \tanh \left[\left(\frac{\beta \pi}{2 \chi}\right) \sqrt{\left(m^{2}+\chi^{2} n^{2}\right)\left(1-\frac{1-2 \nu}{2-2 \nu} \mathrm{Y} \lambda\right)}\right]}-1\right\}
$$

where

$$
Y=\frac{\chi^{2} \beta^{2}}{\pi^{2} 6(1-v)\left(m^{2}+\chi^{2} n^{2}\right)}
$$

As is seen from Table 1, the classical plate theory overestimates the natural frequencies, as expected. When the rotary inertia and the shear effects are taken into account, the difference with the classical theory increases with the vibration mode number. The differences between the Uflyand-Mindlin's original theory and the Uflyand-Mindlin model based on slope inertia are calculated in Table 2.

Moreover, in order to establish the superiority or the inferiority of the modified Uflyand-Mindlin plate models (truncated and based on slope inertia) on the original one, let us compared the results obtained by the modified Uflyand-Mindlin's plate models with the existent results for the original Uflyand-Mindlin plate model applied to a simply supported square plate, found in the literature, by using different numerical methods and the three-dimensional solution. Different models are considered: the Higher Order Shear Deformation Theory (HSDT) given by Shufrin and Eisenberger (2005), the two dimensional Rayleigh-Ritz method (2D Ritz) (Liew, Xiang, \& Kitipornchai, 1993), the three dimensional Rayleigh-Ritz method (Liew, Hung, \& Lim, 1993), the Differential Quadrature Method (DQM) (Stephen, 1997) and the analytical solution from the three dimensional elasticity equations (Wittrick, 1987). The five first natural frequencies, when available, are listed in Table 3. The aim of the numerical method is to confirm the results obtained via Eq. (73). 
Table 1

Comparison of frequency factors for simply supported plates considering the Classical Plate Theory, the Mindlin plate theory and the Mindlin plate theory based on slope inertia.

\begin{tabular}{|c|c|c|c|c|c|c|c|c|c|c|c|c|c|}
\hline \multirow[t]{2}{*}{$\mathrm{m}$} & \multirow[t]{2}{*}{$\mathrm{n}$} & \multicolumn{4}{|l|}{$\chi=1$} & \multicolumn{4}{|l|}{$\chi=2$} & \multicolumn{4}{|l|}{$\chi=5$} \\
\hline & & $\begin{array}{l}\text { Classical } \\
\text { plate } \\
\text { theory }\end{array}$ & $\begin{array}{l}\text { Original } \\
\text { Uflyand- } \\
\text { Mindlin }\end{array}$ & $\begin{array}{l}\text { Truncated } \\
\text { Uflyand- } \\
\text { Mindlin }\end{array}$ & $\begin{array}{l}\text { Slope } \\
\text { inertia } \\
\text { Uflyand- } \\
\text { Mindlin }\end{array}$ & $\begin{array}{l}\text { Classical } \\
\text { plate } \\
\text { theory }\end{array}$ & $\begin{array}{l}\text { Original } \\
\text { Uflyand- } \\
\text { Mindlin }\end{array}$ & $\begin{array}{l}\text { Truncated } \\
\text { Uflyand- } \\
\text { Mindlin }\end{array}$ & $\begin{array}{l}\text { Slope } \\
\text { inertia } \\
\text { Uflyand- } \\
\text { Mindlin }\end{array}$ & $\begin{array}{l}\text { Classical } \\
\text { plate } \\
\text { theory }\end{array}$ & $\begin{array}{l}\text { Original } \\
\text { Uflyand- } \\
\text { Mindlin }\end{array}$ & $\begin{array}{l}\text { Truncated } \\
\text { Uflyand- } \\
\text { Mindlin }\end{array}$ & $\begin{array}{l}\text { Slope } \\
\text { inertia } \\
\text { Uflyand- } \\
\text { Mindlin }\end{array}$ \\
\hline \multirow[t]{5}{*}{1} & 1 & 389.6 & 303.8 & 301.0 & 297.6 & 152.2 & 129.1 & 128.6 & 127.9 & 105.4 & 91.7 & 91.4 & 91.1 \\
\hline & 2 & 2435.2 & 1449.6 & 1402.8 & 1330.8 & 1759.5 & 1111.7 & 1082.4 & 1039.0 & 1589.9 & 1022.3 & 997.0 & 960.1 \\
\hline & 3 & 9740.9 & 4218.7 & 3940.75 & 3420.5 & 8334.6 & 3760.6 & 3529.4 & 3106.3 & 7960.4 & 3634.5 & 3415.7 & 3017.8 \\
\hline & 4 & 28151.2 & 8914.1 & 8038.31 & 6134.9 & 25722.1 & 8383.6 & 7583.7 & 5866.5 & 25061.6 & 8236.0 & 7456.9 & 5790.3 \\
\hline & 5 & 65848.5 & 15584.5 & 13642.3 & 8936.6 & 62104.4 & 15012.1 & 13167.7 & 8730.4 & 61075.7 & 14852.2 & 13035.0 & 8671.9 \\
\hline \multirow[t]{5}{*}{2} & 1 & 2435.2 & 1449.6 & 1402.8 & 1330.8 & 389.6 & 303.8 & 301.0 & 297.6 & 131.1 & 112.4 & 111.9 & 111.5 \\
\hline & 2 & 6234.2 & 3025.3 & 2863.0 & 2578.1 & 2435.2 & 1449.6 & 1402.8 & 1330.8 & 1685.7 & 1073.1 & 1045.5 & 1005.0 \\
\hline & 3 & 16462.1 & 6151.9 & 5650.5 & 4638.8 & 9740.9 & 4218.7 & 3940.7 & 3420.5 & 8173.2 & 3706.4 & 3480.6 & 3068.4 \\
\hline & 4 & 38963.6 & 11082.0 & 9880.0 & 7152.4 & 28151.2 & 8914.1 & 8038.3 & 6134.9 & 25438.0 & 8320.3 & 7529.4 & 5833.9 \\
\hline & 5 & 81921.0 & 17896.1 & 15549.7 & 9717.4 & 65848.5 & 15584.5 & 13642.3 & 8936.6 & 61662.4 & 14943.5 & 13110.9 & 8705.4 \\
\hline \multirow[t]{5}{*}{3} & 1 & 9740.9 & 4218.7 & 3940.7 & 3420.5 & 1028.9 & 709.1 & 695.9 & 677.8 & 180.2 & 150.9 & 150.1 & 149.3 \\
\hline & 2 & 16462.1 & 6151.9 & 5650.5 & 4638.8 & 3805.0 & 2067.9 & 1981.9 & 1841.1 & 1851.7 & 1159.5 & 1127.9 & 1080.8 \\
\hline & 3 & 31560.5 & 9629.0 & 8648.4 & 6483.9 & 12328.3 & 5006.2 & 4642.0 & 3936.7 & 8534.0 & 3827.0 & 3589.2 & 3152.5 \\
\hline & 4 & 60880.7 & 14821.8 & 13009.8 & 8660.7 & 32443.3 & 9809.0 & 8801.5 & 6569.6 & 26071.5 & 8461.1 & 7650.2 & 5906.2 \\
\hline & 5 & 112604.9 & 21812.8 & 18754.1 & 10879.0 & 72332.3 & 16543.6 & 14435.4 & 9270.2 & 62646.7 & 15095.9 & 13237.3 & 8761.0 \\
\hline \multirow[t]{5}{*}{4} & 1 & 28151.2 & 8914.1 & 8038.3 & 6134.9 & 2435.2 & 1449.6 & 1402.8 & 1330.8 & 262.0 & 212.5 & 211.0 & 209.3 \\
\hline & 2 & 38963.6 & 11082.0 & 9880.0 & 7152.4 & 6234.2 & 3025.3 & 2863.0 & 2578.1 & 2097.2 & 1284.0 & 1246.1 & 1189.0 \\
\hline & 3 & 60880.7 & 14821.8 & 13009.8 & 8660.7 & 16462.1 & 6151.9 & 5650.5 & 4638.8 & 9052.2 & 3997.3 & 3742.3 & 3270.0 \\
\hline & 4 & 99746.9 & 20237.8 & 17469.1 & 10433.8 & 38963.6 & 11082.0 & 9880.0 & 7152.4 & 26971.6 & 8658.8 & 7819.8 & 6006.7 \\
\hline & 5 & 163744.7 & 27395.1 & 23277.2 & 12261.0 & 81921.0 & 17896.1 & 15549.7 & 9717.4 & 64037.7 & 15309.4 & 13414.4 & 8838.2 \\
\hline \multirow[t]{5}{*}{5} & 1 & 88439.7 & 15584.5 & 13642.3 & 8936.6 & 5120.1 & 2603.5 & 2476.9 & 2260.8 & 389.6 & 303.8 & 301.0 & 297.6 \\
\hline & 2 & 122407.9 & 17896.1 & 15549.7 & 9717.4 & 10234.0 & 4373.9 & 4079.5 & 3524.5 & 2435.2 & 1449.6 & 1402.8 & 1330.8 \\
\hline & 3 & 191262.3 & 21812.8 & 18754.1 & 10879.0 & 22653.7 & 7684.6 & 6982.1 & 5499.9 & 9740.9 & 4218.7 & 3940.7 & 3420.5 \\
\hline & 4 & 313364.2 & 27395.1 & 23277.2 & 12261.0 & 48223.6 & 12748.1 & 11280.8 & 7858.9 & 28151.2 & 8914.1 & 8038.3 & 6134.9 \\
\hline & 5 & 514419.1 & 34688.4 & 29132.2 & 13712.8 & 95126.1 & 19649.9 & 16988.3 & 10260.3 & 65848.5 & 15584.5 & 13642.3 & 8936.6 \\
\hline
\end{tabular}

Table 2

Percentage difference between of simply supported rectangular plates considering the Uflyand-Mindlin plate theory and the Uflyand-Mindlin plate theory based on slope inertia.

\begin{tabular}{ccccc}
\hline \multirow{2}{*}{$m$} & $n$ & \multicolumn{2}{c}{ Relative error (\%) } & \\
\cline { 3 - 5 } & & $\chi=1$ & $\chi=2$ & $\chi=5$ \\
\hline 1 & 1 & 2.04 & 0.91 & 0.66 \\
& 2 & 8.19 & 6.54 & 6.09 \\
& 3 & 18.92 & 17.40 & 16.97 \\
& 4 & 31.18 & 30.02 & 29.69 \\
2 & 5 & 42.66 & 41.84 & 41.61 \\
& 1 & 8.19 & 2.04 & 0.80 \\
& 2 & 14.78 & 8.19 & 6.35 \\
& 3 & 24.60 & 18.92 & 17.21 \\
& 4 & 35.46 & 31.18 & 29.88 \\
3 & 5 & 45.70 & 42.66 & 41.74 \\
& 1 & 18.92 & 4.42 & 1.06 \\
& 2 & 24.60 & 10.97 & 6.78 \\
& 3 & 32.66 & 21.37 & 17.62 \\
& 4 & 41.57 & 33.02 & 30.20 \\
4 & 5 & 50.13 & 43.97 & 41.96 \\
& 1 & 31.18 & 8.19 & 1.46 \\
& 2 & 35.46 & 14.78 & 7.40 \\
& 3 & 41.57 & 24.60 & 18.20 \\
& 4 & 48.44 & 35.46 & 30.63 \\
5 & 5 & 55.24 & 45.70 & 42.27 \\
& 1 & 42.66 & 13.16 & 2.04 \\
& 2 & 45.70 & 19.42 & 8.19 \\
& 3 & 50.13 & 28.43 & 18.92 \\
& 3 & 55.24 & 38.35 & 31.18 \\
& 4 & 60.47 & 47.78 & 42.66 \\
\hline
\end{tabular}


Table 3

Comparison study of frequency parameters for an all edges simply supported squared plate.

\begin{tabular}{|c|c|c|c|c|c|c|c|c|c|}
\hline$\beta$ & $\begin{array}{l}\text { 3D analytical } \\
\text { (Wittrick, } \\
\text { 1987) }\end{array}$ & $\begin{array}{l}\text { Classical } \\
\text { plate theory } \\
\text { Leissa }\end{array}$ & $\begin{array}{l}\text { Original } \\
\text { Uflyand- } \\
\text { Mindlin }\end{array}$ & $\begin{array}{l}\text { Truncated } \\
\text { Uflyand- } \\
\text { Mindlin }\end{array}$ & $\begin{array}{l}\text { Slope inertia } \\
\text { Uflyand- } \\
\text { Mindlin }\end{array}$ & $\begin{array}{l}\text { HSDT } \\
\text { (Shufrin \& } \\
\text { Eisen- } \\
\text { berger, 2005) }\end{array}$ & $\begin{array}{l}\text { 2D Ritz Liew } \\
\text { (Liew et al., } \\
\text { 1998) }\end{array}$ & $\begin{array}{l}\text { 3D Ritz } \\
\text { (Liew et al., } \\
\text { 1993) }\end{array}$ & $\begin{array}{l}\text { 3D DQM } \\
\text { (Malik \& } \\
\text { Bert, 1998) }\end{array}$ \\
\hline \multirow[t]{5}{*}{0.001} & 389.6 & 389.6 & 389.6 & 389.6 & 389.6 & - & 389.6 & - & - \\
\hline & 2435.1 & 2435.2 & 2435.2 & 2435.2 & 2435.2 & - & 2435.2 & - & - \\
\hline & 6233.8 & 6234.0 & 6234.0 & 6234.0 & 6234.0 & - & 6234.0 & - & - \\
\hline & 9740.4 & 9740.6 & 9740.6 & 9740.8 & 9740.7 & - & 9740.7 & - & - \\
\hline & 16461.2 & 16461.3 & 16461.3 & 16461.5 & 16461.3 & - & 16461.7 & - & - \\
\hline \multirow[t]{5}{*}{0.1} & 357.0 & 389.6 & 364.2 & 369.9 & 363.6 & 363.5 & - & 364.4 & 364.4 \\
\hline & 2080.1 & 2435.2 & 2077.9 & 2144.5 & 2059.8 & 2069.1 & - & 2081.1 & 2081.1 \\
\hline & 4914.4 & 6234.0 & 4903.0 & 5122.9 & 4806.7 & 4873.4 & - & 4914.5 & 4914.6 \\
\hline & 7307.8 & 9740.6 & 7287.3 & 7663.1 & 7080.7 & 7235.9 & - & 7308.1 & 7308.2 \\
\hline & 11528.1 & 16461.3 & 11487.0 & 12171.9 & 10996.1 & 11392.4 & - & 11528.5 & 11528.2 \\
\hline \multirow[t]{5}{*}{0.2} & 306.6 & 389.6 & 306.4 & 320.2 & 300.4 & 304.6 & - & 307.2 & 307.2 \\
\hline & 1480.2 & 2435.2 & 1473.4 & 1578.9 & 1355.9 & 1458.4 & - & 1480.9 & 1480.9 \\
\hline & 2414.9 & 6234.0 & 3089.8 & 3337.9 & 2642.5 & 3058.0 & - & 3112.2 & 3112.2 \\
\hline & 3111.9 & 9740.6 & 4319.0 & 4672.9 & 3516.1 & 4265.8 & - & 4355.5 & 4355.5 \\
\hline & 4355.4 & 16461.3 & 6316.6 & 6830.9 & 4784.6 & 6238.9 & - & - & - \\
\hline
\end{tabular}

In their paper, Liew et al. (1993) considered thickness ratios equal to $0.1,0.2,0.3$ and 0.5 . $\beta$ is equal to 0.3 or 0.5 is too high. Consequently, these results are not relevant for plates for which the thickness has to be very small compared to the lengths and will be ignored here. That is why we retain only results for a thickness ratio smaller than 0.2 .

First of all, the nondimensional natural frequencies calculated by using Eq. (73) for the original Uflyand-Mindlin plate are confirmed by those obtained by using the 3D Ritz method (Liew et al., 1993) and the differential quadrature method (Malik \& Bert, 1998), even if these numerical methods trend to overestimate the natural frequencies.

For small modes of frequencies, the results almost coincide between the different versions of the Uflyand-Mindlin plate model (original, truncated and based on slope inertia). The truncated and original Uflyand-Mindlin's plate models provide close results for any thickness ratio. It appears that the Uflyand-Mindlin's plate model based on slope inertia gives lower non dimensional natural frequencies than the two other models for any thickness ratio and mode of frequency.

The higher order-shear deformation theory, naturally more accurate than the first-order shear deformation theory, provides results close to those obtained by the original Uflyand-Mindlin plate theory. It trends to confirm the superiority of the original Uflyand-Mindlin plate theory on the two other engineering models. However, it is seen, according to the exact three dimensional solution, that the Uflyand-Mindlin's plate model based on slope inertia, for high frequencies, is much better than the traditional Mindlin's plate model or its truncated version. Furthermore, whereas the original Uflyand-Mindlin plate theory predicts two branches of frequencies, truncated version and the one based on slope inertia lead to only one branch of natural frequencies. Consequently, using the three-dimensional model as a reference model, it appears that the Uflyand-Mindlin's plate model based on slope inertia provide more accurate results than the two other versions of the Uflyand-Mindlin theory. For any considered case, the difference between the models is relatively small. The truncated Uflyand-Mindlin's plate model and the version based on slope inertia present the advantage to lead to only one branch of natural frequencies.

\section{Conclusion}

This paper presents derivations of the different Uflyand-Mindlind models; it is also a guide that compares them and provides arguments of different nature to prefer one model over the other. Each of them has some advantages and disadvatages. The original Uflyand-Mindlind plate model is derivable both from the equilibrium equations and variationally. However, it overcorrects the shear deformation effect. The truncated Uflyand-Mindlind model does not overestimate this effect and is asymptotically consistent. Nevertheless, it cannot be obtained variationally. The last model presented, the Uflyand-Mindlin plate model based on slope inertia, as the truncated model, does not overestimate the shear effect but it is not derivable from the equilibrium equations but rather variationally.

Furthermore, as explained by Stephen (1997), the original Uflyand-Mindlin plate model predicts more than one frequency branch. The second branch is judged by Stephen "meaningless" and is "a consequence of an otherwise remarkable approximate engineering theory". This paper does not go so far as to identify the second branch of frequencies as "meaningless". Nevertheless, it is worth noting that the truncated Uflyand-Mindlin plate model and the Uflyand-Mindlin plate model based on slope inertia do not predict this second branch of frequencies. In this sense they are not overcomplicated theories, as the original Uflyand-Mindlin's plate theory appears to constitute to some investigators.

To sum up, in this study, the Uflyand-Mindlin plate equations has been derived through different approaches. Asymptotically, at the second order, it leads to the truncated version, also obtainable by a correction of the equations of motion. Variationally, it has been proposed to use a modified expression of the kinetic energy. This results in a governing differential 
equation which contains an additional sixth order derivative term but in which a fourth order time derivative - that is characteristic to the original Uflyand-Mindlin plate theory - does not appear. The comparison of contributions of each term into the equation shows that the fourth order time derivative term can be neglected for big aspect ratios and small vibration mode numbers. In this case, the suggested theory is associated with a governing differential simpler equation than the original Uflyand-Mindlin equation.

It appears that additional studies are needed to ascertain the region of validity of the suggested theory for isotropic, orthotropic, and anisotropic plates. Papers on the above topics are underway and will be published elsewhere.

\section{Acknowledgement}

\section{Authors of this work thank Professor Mark Kachanov for making constructive suggestions.}

\section{References}

Berdichevsky, V. L. (1973). Dynamic theory of thin elastic plates. Izv. AN SSSR Mekhanika Tverdogo Tela, 8(6), 99-109.

Berdichevsky, V. L., \& Kvashina, S. S. (1974). On equations describing the transverse vibrations of elastic bars. Journal of Applied Mathematics and Mechanics, 104-119.

Bresse, J. (1859). Cours de mécanique appliquée. Paris: Mallet-Bachelier.

Brunelle, E. J. (1971). Buckling of transversely isotropic Mindlin plates. AIAA Journal, 9, 1018-1022.

Brunelle, E. J., \& Roberts, S. R. (1974). Initially stressed Mindlin plates. AIAA Journal, 12, 1036-1045.

Cauchy, A. L. (1828). Sur l'équilibre et le mouvement d'une plaque solide. Exercices Math, 3, 328 (in French).

Elishakoff, I. (1994). Generalization of the Bolotin's dynamic edge-effect method for vibration analysis of Mindlin plates. In JM Cuschieri, SAL Glegg, \& DM Yeager (Eds.), NOISE-CON 94. Proceedings of the 1994 national conference on noise control engineering; 1994 May 01-04; Fort Lauderdale (pp. 911-916).

Elishakoff, I. (2009). An equation both more consistent and simpler than Bresse-Timoshenko equation. In R. Gilat, \& L. Sills-Banks (Eds.), Advances in mathematical modeling and experimental methods for materials and structures (pp. 249-254). Berlin: Springer Verlag.

Elishakoff, I., Kaplunov, J., \& Nolde, E. (2015). Celebrating the centenary of Timoshenko's study of effects of shear deformation and rotary inertia. Applied Mechanics Reviews, 67 article 060802-1.

Elishakoff, I., \& Livshits, D. (1984). Some closed-form solutions in random vibration of Bernoulli-Euler beams. International Journal of Engineering Science, 22, $1291-1301$.

Elishakoff, I., \& Lubliner, E. (1985). Random vibration of a structure via classical and nonclassical theories. In S. Eggwertz (Ed.), Probabilistic methods in mechanics and structures (pp. 455-468). Springer-Verlag: Berlin.

Elishakoff, I., Pentaras, D., Dujat, K., Versaci, C., Muscolino, G., Storch, J., et al. (2012). Carbon nanotubes and nanosensors: Vibrations, buckling and ballistic impact. London: ISTE - Wiley.

Falsone, G., Settineri, D., \& Elishakoff, I. (2014). A new locking-free finite element method based on more consistent version of Mindlin plate equation. Archive of Applied Mechanics, 84, 967-983.

Falsone, G., Settineri, D., \& Elishakoff, I. (2015). A new class of interdependent shape polynomials for the FE dynamic analysis of Mindlin plate Timoshenko beam. Archive of Applied Mechanics, 50, 767-780.

Germain, S. (1826). Remarques sur la nature, les bornes et l'étendue de la question des surfaces élastiques et équation générale de ces surfaces Paris. (in French).

Goldenveizer, A. L., Kaplunov, J. D., \& Nolde, E. V. (1993). On Timoshenko-Reissner type-theories of plates and shells. International Journal of Solids and Structures, 30(5), 675-694.

Herrmann, G. (1974). R.D. Mindlin and applied mechanics. New York: Pergamon.

Hutchinson, J. R. (1984). Vibrations of thick free circular plates, exact versus approximate solutions. Journal of Applied Mechanics, 51, 581-585.

Kaneko, T. (1975). On Timoshenko's correction for shear in vibrating beams. Journal of Physics D: Applied Physics, 8, 1927-1939.

Kaplunov, J. D. (1996). Asymptotic approximations of the 3D dybnamical equations of elasticity for the case of a thin body. Zeitschrift für angewandte Mathematik und Mechanik, 76, 237-238.

Kirchhoff, G. (1850). Über das Gleichgewicht und die Bewegung einer elastichen Scheibe. Journal für die reine und angewandte Mathematik, 40, 51-58.

Lagrange, J. L. (1828). Note à propos du mémoire de Sophie Germain pour le prix de l'Académie. Annales de Chimie et de Physique, 39, 149-207 (in French). Leissa, A. W. (1969). Vibrations of plates. NASA. Washington: NASA SP-160, Office of Technology Utilization.

Liew, K. M., Hung, K. C., \& Lim, M. K. (1993). A continuum three-dimensional vibration analysis of thick rectangular plates. International Journal of Solids and Structures, 30, 3357-3379.

Liew, K. M., Xiang, Y., \& Kitipornchai, S. (1993). Transverse vibration of thick rectangular plates - I. [1]Comprehensive sets of boundary conditions. Computers and Structures, 49, 1-29.

Liew, K. M., Xiang, Y., Kitipornchai, S., \& Wang, C. M. (1998). Vibration of Mindlin plates: Programming the p-version Ritz method. Oxford: Elsevier.

Malik, M., \& Bert, C. W. (1998). Three-dimensional elasticity solutions for free vibrations of rectangular plates by the differential quadrature method. International Journal of Solids and Structures, 35, 299-318.

Mindlin, R. D. (1951). Influence of rotary inertia and shear on flexural motions of isotropic, elastic plates. Journal of Applied Mechanics, 18, 31-38.

Mindlin, R. D., Schacknow, A., \& Dereciewicz, H. (1956). Flexural vibrations of rectangular plates. Journal of Applied Mechanics, 23, 430-436.

Navier, C. L. M. H. (1823). Bulletin des Sciences de la Société Philomathique de Paris (in French).

Poisson, S. D. (1829). Mémoires Sur l'équilibre et le mouvement des corps élastiques. Mémoire de l'Académie des Sciences, 8, 357-571 (in French).

Rayleigh, J. W. (1877). Theory of sound: Vol. 1. London: Macmillan.

Reddy, J. N. (2006). Theory and analysis of plates and shells (2nd ed.). CRC Press.

Reismann, H. (1988). Elastic plates, theory and application. New York: John Wiley \& Sons.

Reissner, E. (1944). On the theory of elastic plates. Journal of Mathematics and Physics, 23, 184-191.

Reissner, E. (1945). The effect of transverse shear deformation on the bending of elastic plates. Journal of Applied Mechanics, 12 , A69-A77.

Sharma, A., Sharda, H. B., \& Nath, Y. (2005). Stability and vibration of Mindlin sector plates: An analytical approach. AIAA Journal, 4, $1109-1116$.

Shufrin, I., \& Eisenberger, M. (2005). Stability and vibration of shear deformable plates - first order and higher order analysis. International Journal of Solids and Structures, 42, 1225-1251.

Stephen, N. G. (1981). Considerations on second order beam theories. International Journal of Solids and Structures, 17, 325-333.

Stephen, N. G. (1997). Mindlin plate theory: Best shear coefficient and higher spectra validity. Journal of Sound and Vibration, 202(4), 539-553.

Timoshenko, S. P. (1921). On the correction for shear of the differential equation for transverse vibrations of prismatic bars. Philosophical Magazine, 41, 744-746.

Timoshenko, S. P. (1922). On the transverse vibration of bars with uniform cross-section. Philosophical Magazine, 43, 125-131.

Uflyand, Y. S. (1948). The propagation of waves in the transverse vibrations of bars and plates. PMM= Prikl. Math. Mech, 12, $287-461$.

Vashakmadze, T. S. (1999). The theory of anisotropic elastic plates. Dordrecht: Kluwer Academic Publishers.

Ventsel, E., \& Krauthammer, T. (2001). Thin plates and shells. New York: Marcel Dekker, Inc.

Wang, C. M. (1994). Natural frequencies formula for simply supported mindlin plates. Journal of Vibration and Acoustics, 116, 536-540. 
Wang, C. M., Reddy, J. N., \& Lee, K. H. (2000). Shear deformable beams and plates: Relationships with classical solutions. Oxford (UK): Elsevier.

Widera, O. E. (1970). An asymptotic theory for the motion of elastic plates. Acta Mechanica, 9, 54-66.

Wittrick, W. H. (1987). Analytial, three-dimensional elasticity solutions to some plate problems, and some observations on Mindlin's plate theory. International Journal of Solids and Structures, 23, 441-464. 